\title{
Dynamically assisted tunneling in the impulse regime
}

\author{
Christian Kohlfürst, ${ }^{1}$ Friedemann Queisser $\odot,{ }^{1,2}$ and Ralf Schützhold ${ }^{1,2}$ \\ ${ }^{1}$ Helmholtz-Zentrum Dresden-Rossendorf, Bautzner Landstraße 400, 01328 Dresden, Germany \\ ${ }^{2}$ Institut für Theoretische Physik, Technische Universität Dresden, 01062 Dresden, Germany
}

(Received 27 April 2021; accepted 15 July 2021; published 13 August 2021)

\begin{abstract}
We study the enhancement of tunneling through a potential barrier $V(x)$ by a time-dependent electric field with special emphasis on pulse-shaped vector potentials such as $A_{x}(t)=A_{0} / \cosh ^{2}(\omega t)$. In addition to the known effects of preacceleration and potential deformation already present in the adiabatic regime, as well as energy mixing in analogy to the Franz-Keldysh effect in the nonadiabatic (impulse) regime, the pulse $A_{x}(t)$ can enhance tunneling by "pushing" part of the wave function out of the rear end of the barrier. Aside from the natural applications in condensed matter and atomic physics, these findings could be relevant for nuclear fusion, where pulses $A_{x}(t)$ with $\omega=1 \mathrm{keV}$ and peak field strengths of $10^{16} \mathrm{~V} / \mathrm{m}$ might enhance tunneling rates significantly.
\end{abstract}

DOI: 10.1103/PhysRevResearch.3.033153

\section{INTRODUCTION}

One of the most striking differences between classical and quantum mechanics is the tunnel effect. Even though this phenomenon cannot be directly observed with the naked eye [1], it plays an important role in many areas of physics and across a wide variety of length scales, including ultracold atoms in optical lattices on the micrometer scale (see, e.g., $[2,3]$ ), and ranging down to nuclear physics in the femtometer regime [4-6]. However, although tunneling is usually taught in the first lecture course on quantum mechanics, our intuition and understanding, especially regarding time-dependent scenarios, is still far from complete [7].

In order to be more specific, let us consider the onedimensional (1D) Schrödinger equation describing a particle with energy $E$ and mass $m$ incident on a potential barrier $V(x)$. Then, using the standard WKB approximation, we may derive the tunneling probability $\mathcal{P}$ (or, more precisely, its exponential contribution) and arrive at the following famous expression, which, as Coleman [8] puts it, "every child knows" $(\hbar=1)$

$$
\mathcal{P} \sim e^{-2 \mathfrak{S}_{E}}=\exp \left\{-2 \int_{x_{\mathrm{in}}^{E}}^{x_{\mathrm{out}}^{E}} d x \sqrt{2 m[V(x)-E]}\right\} .
$$

Here, $x_{\text {in }}^{E}$ and $x_{\text {out }}^{E}$ are the classical turning points with $V\left(x_{\text {in }}^{E}\right)=$ $V\left(x_{\text {out }}^{E}\right)=E$ (assuming that there are only two of them). Alternatively, one could employ the instanton picture where $\mathfrak{S}_{E}$ denotes the Euclidean action [8].

Adding a temporal dependence $V(t, x)$, however, the situation becomes far more complex. Only in certain limiting cases, such as slowly varying or rapidly oscillating potentials

Published by the American Physical Society under the terms of the Creative Commons Attribution 4.0 International license. Further distribution of this work must maintain attribution to the author(s) and the published article's title, journal citation, and DOI.
$V(t, x)$, simple expressions similar to Eq. (1) can be derived via the quasistatic or the time-averaged potential approximation.

In the following, we do not consider the general case $V(t, x)$, but we focus on the simpler (yet nontrivial) scenario of a static potential barrier superimposed by a purely timedependent electric field. In the Coulomb gauge, this case can be represented by a space-time-dependent potential $V(t, x)=$ $V_{0}(x)+x V_{1}^{\prime}(t)$, but we find it more convenient to represent the electric field via the vector potential $A(t)$ in the temporal gauge.

In order to understand the impact of the additional electric field on the tunneling probability (1) of a charged particle, let us try to separate the phases of the temporal evolution:

First, even before hitting the barrier, the electric field can accelerate (or decelerate) the particle which effectively increases (or decreases) the energy $E$ and changes the classical turning points $x_{\text {in }}^{E}$ and $x_{\text {out }}^{E}$. This effect already occurs in the adiabatic regime of slowly varying electric fields.

In contrast, the time dependence of $A(t)$ can induce additional phenomena in the nonadiabatic (impulse) regime. Thus, as the second effect, the energy $E$ is no longer conserved due to this temporal variation, i.e., it can effectively shift the energy up or down. Assuming a harmonic oscillation $A(t)=A_{0} \sin (\omega t)$, for example, this effect can be understood in the Floquet picture $[9,10]$ where we get side bands with effective energies $E \pm n \hbar \omega$ (in analogy to the Franz-Keldysh effect $[11,12]$.) In view of the exponential decay of the wave function inside the barrier, this effect can typically most efficiently enhance the tunneling rate when the mixing occurs near the front end $x_{\text {in }}^{E}$ of the barrier.

Third, the electric field effectively deforms the barrier and thereby changes the tunneling probability (1). Similar to the first contribution (preacceleration) and in distinction to the second (energy mixing), this effect already occurs in the adiabatic regime.

Finally, the time-dependent electric field may enhance tunneling by effectively "pushing" part of the wave function out 
of the rear end $x_{\text {out }}^{E}$ of the barrier in the nonadiabatic (impulse) regime. (Reversing the electric field would then suppress tunneling by "pulling" it back into the barrier.) In the following, we shall study this fourth displacement effect and its relation to the other three contributions in more detail.

Of course, the electric field does also influence the wave packet after tunneling through the barrier, but this does not change the tunneling probability, unless the wave packet is "pulled" back into the barrier again. This case is relevant for scenarios where the time-averaged potential approximation applies, but it will not play a role here.

One should also stress that the above dissection according to the various stages of the temporal evolution (or the spatial regions) is not sharp as we are dealing with wave packets instead of point particles. Due to the corresponding uncertainty, all these phenomena will be intertwined in general. Only in appropriate limiting cases, it will be possible to separate these effects clearly, as we shall see below.

\section{Outline}

In order to establish the basic mechanisms underlying our main ideas, we briefly recapitulate the Kramers-Henneberger transformation in Sec. II. Based on this mapping, we derive and discuss approximate analytic solutions for rectangular and triangular potential barriers in Secs. III and IV. Albeit idealized, these simple toy models allow us to identify and to distinguish the various enhancement effects mentioned above. Comparing the rectangular potential with the two possible orientations of the triangular barrier, for example, we may study the dependence of those effects (especially energy mixing and displacement) on the potential slope at the turning points $x_{\text {in }}^{E}$ and $x_{\text {out }}^{E}$. In Sec. $\mathrm{V}$, we then briefly discuss potential experimental realizations, before considering the case of nuclear fusion in Sec. VI in more detail. Apart from a summary of our main results, Sec. VII contains a brief discussion of the prospects for experimental applications. Finally, the extension to further scenarios such as muon-assisted fusion is discussed in Sec. VIII.

\section{KRAMERS-HENNEBERGER MAP}

The fourth displacement effect described above can be nicely understood using the Kramers-Henneberger transformation [13] which describes an exact mapping of a purely time-dependent electric field to the induced quiver motion $\chi(t)$. In order to briefly recapitulate the basic principle, let us start from the Schrödinger equation

$$
i \partial_{t} \psi=-\frac{[\nabla-i q \boldsymbol{A}(t)]^{2}}{2 m} \psi+V \psi .
$$

For a purely time-dependent vector potential $\boldsymbol{A}(t)$, we may eliminate the quadratic term $q^{2} A^{2} /(2 m)$ by a global phase transformation $\psi \rightarrow e^{i \phi(t)} \psi$ with $\dot{\phi}=q^{2} A^{2} /(2 m)$.

If we now consider a Galilei transformation $\boldsymbol{r} \rightarrow \boldsymbol{r}+\boldsymbol{v} t$ which changes the time derivative as $\partial_{t} \rightarrow \partial_{t}-\boldsymbol{v} \cdot \boldsymbol{\nabla}$, we see that the additional term is equivalent to a constant vector potential $\boldsymbol{A}$ proportional to the velocity $\boldsymbol{v}$. This identification also works for time-dependent vector potentials $\boldsymbol{A}(t)$ which can thus be represented by the displacement $\boldsymbol{r} \rightarrow \boldsymbol{r}+\chi(t)$ given by

$$
\dot{\chi}(t)=-\frac{q A(t)}{m},
$$

which is precisely the classical solution of a point particle with mass $m$ and charge $q$ in the electric field generated by the vector potential $\boldsymbol{A}(t)$. Thus, the vector potential $\boldsymbol{A}(t)$ can be translated into a corresponding displacement $\chi(t)$ of the wave function $\psi$, i.e., $\psi[t, \boldsymbol{r}] \rightarrow \psi[t, \boldsymbol{r}-\chi(t)]$. Turning this argument around, the Schrödinger equation (2) in the presence of a static potential barrier $V(\boldsymbol{r})$ and a purely time-dependent electric field $\boldsymbol{A}(t)$ can be mapped onto a Schrödinger equation without any vector potential $\boldsymbol{A}=0$, but with a quivering potential barrier $V_{0}[\boldsymbol{r}] \rightarrow V_{\chi}[t, \boldsymbol{r}]=V_{0}[\boldsymbol{r}+\chi(t)]$.

\section{RECTANGULAR POTENTIAL}

In order to study the four effects (preacceleration, energy mixing, potential deformation, and displacement) mentioned in the Introduction, let us start with the extremely simple case of a rectangular (box) potential of height $V_{0}$ and length $L$ in one dimension

$$
V(x)=V_{0} \Theta(x) \Theta(L-x) .
$$

In the absence of the vector potential $A(t)$, we would have an incident wave with energy $E_{\text {in }}$ on the left-hand side $x<0$ of the potential, plus a reflected wave with the same energy $E_{\text {in }}$. Including the vector potential $A(t)$ changes the incident wave according to the Kramers-Henneberger transformation described above, while the reflected wave will in general contain a mixture of different energies $E$. Thus, we arrive at the general ansatz

$$
\begin{aligned}
\psi(t, x<0)= & e^{-i E_{\mathrm{in}} t+i \sqrt{2 m E_{\mathrm{in}}}[x-\chi(t)]} \\
& +\int d E \psi_{\mathrm{ref}}(E) e^{-i E t-i \sqrt{2 m E}[x-\chi(t)]} .
\end{aligned}
$$

The term $\sqrt{2 m E_{\text {in }}} \chi(t)$ in the exponent of the first line describes the acceleration by the electric field before hitting the barrier. Similarly, we use the general ansatz for the solutions inside the barrier

$$
\begin{aligned}
\psi(t, 0<x<L)= & \int d E e^{-i E t} \\
& \times\left(\psi_{\text {int }}^{+}(E) e^{+\sqrt{2 m\left(V_{0}-E\right)}[x-\chi(t)]}\right. \\
& \left.+\psi_{\text {int }}^{-}(E) e^{-\sqrt{2 m\left(V_{0}-E\right)}[x-\chi(t)]}\right),
\end{aligned}
$$

as well as the transmitted solutions

$$
\psi(t, x>L)=\int d E \psi_{\operatorname{tra}}(E) e^{-i E t+i \sqrt{2 m E}[x-\chi(t)]},
$$

where we have assumed that there is no wave incident from the right-hand side of the barrier $x>L$. Without the additional electric field, $\psi_{\text {tra }}(E)$ would just be the energydependent transmission amplitude of the static box potential (4) multiplied by the incident wave function in energy space. Modifications of $\psi_{\operatorname{tra}}(E)$ induced by that electric field, i.e., $\chi(t)$, then correspond to the enhancement (or suppression) of tunneling.

The matching conditions for $\psi(t, x)$ and $\psi^{\prime}(t, x)$ at $x=0$ and $L$ for all times $t$ uniquely determine $\psi_{\text {ref }}(E), \psi_{\text {int }}^{ \pm}(E)$, and 
$\psi_{\text {tra }}(E)$. While the resulting set of equations is linear and thus solvable via numerical discretization, for example, we shall use some analytical approximations to gain further inside in the following.

\section{A. Sudden approximation}

As our first example, let us consider a very strong and short pulse $A(t)$ which can be approximated by a Dirac delta function $A(t) \propto \delta(t)$. According to Eq. (3), this corresponds to a sudden displacement $\chi(t)=\Delta \chi \Theta(t)$, where we assume $L>\Delta \chi>0$. Considering an initially stationary solution with $E_{\text {in }}$, we find two major effects. At the front of the barrier (around $x=0$ ), the displaced wave function is no longer an energy eigenstate, implying a mixture of energies $E$. The contributions corresponding to higher energies $E>E_{\text {in }}$ can then tunnel through the barrier more easily than the initial stationary solution with $E_{\text {in }}$.

At the rear of the barrier (around $x=L$ ), a part of the exponential tunneling tail with length $\Delta \chi$ is "pushed" out of the barrier and thus the probability density behind the barrier is exponentially enhanced by a factor of $e^{2 \sqrt{2 m\left(V_{0}-E_{\text {in }}\right)}} \Delta \chi$, which could be quite large.

\section{B. Opaque-barrier approximation}

In order to treat more realistic time dependencies $\chi(t)$, we employ the opaque-barrier (low-energy) approximation. To this end, we assume that the potential height $V_{0}$ is much larger than all other energy and frequency scales, such as $V_{0} \gg E_{\text {in }}$. Furthermore, we assume that the barrier width $L$ is also much larger than all other length scales, such as $L \gg|\chi|$. As a result, the tunneling rate is strongly suppressed due to $\sqrt{2 m V_{0}} L \gg 1$, i.e., the barrier is very opaque (see also [14]).

In addition to the large quantity $\sqrt{2 m V_{0}} L \gg 1$, we only keep those terms where an energy $E \ll V_{0}$ is combined with the long length $L$ as well as those terms where the displacement $\chi \ll L$ is combined with the large barrier height $V_{0}$. To be consistent, we neglect all terms which do not contain a large quantity ( $V_{0}$ or $L$ ), such as all combinations of an energy $E \ll V_{0}$ with a displacement $\chi \ll L$. Within this scheme, the acceleration before the barrier $\sqrt{2 m E_{\text {in }}} \chi(t)$ in Eq. (5) is neglected.

Using this approximation scheme, we may simplify the matching conditions at $x=0$ and $L$. Due to the exponential suppression of the tunneling rate, we have $\psi_{\text {int }}^{+}(E) \lll \psi_{\text {int }}^{-}(E)$ such that the contribution from $\psi_{\text {int }}^{+}(E)$ can be neglected at $x=0$. To leading order in $E / V_{0}$, we then find $\psi_{\text {ref }}(E) \approx$ $-\delta\left(E-E_{\text {in }}\right)$ which yields

$$
\psi_{\mathrm{int}}^{-}(E) \approx-2 i \sqrt{\frac{E_{\mathrm{in}}}{V_{0}}} \int \frac{d t}{2 \pi} e^{i\left(E-E_{\mathrm{in}}\right) t-\sqrt{2 m V_{0}} \chi(t)} .
$$

Consistent with the simple picture described in the previous Sec. III A, this equation describes the energy mixing at the front of the barrier $x=0$ due to the time-dependent displacement $\chi(t)$. Then we may use the remaining matching condition at $x=L$ in order to determine the transmitted solution

$$
\psi_{\mathrm{tra}}(E) \approx \psi_{E}^{0} \int \frac{d t}{2 \pi} e^{i\left(E-E_{\mathrm{in}}\right) t-\sqrt{2 m V_{0}}[\chi(t+i \mathfrak{T})-\chi(t)]},
$$

where $\psi_{E}^{0}=4 e^{-\sqrt{2 m V_{0}} L+E_{\text {in }} \mathfrak{T}-i \sqrt{2 m E} L} \sqrt{E_{\text {in }} / V_{0}}$ collects the $\chi$ independent factors and $\mathfrak{T}$ denotes the Büttiker-Landauer traversal time [14] for this case (with $V_{0} \gg E_{\text {in }}$ )

$$
\mathfrak{T}=L \sqrt{\frac{m}{2 V_{0}}} .
$$

Note that the first two exponentials $e^{-\sqrt{2 m V_{0}} L+E_{\text {in }} \mathfrak{T}}$ in $\psi_{E}^{0}$ are the leading-order contributions of the undisturbed tunneling exponent $e^{-\sqrt{2 m\left(V_{0}-E_{\text {in }}\right)} L}$.

It is illuminating to see how the Büttiker-Landauer traversal time $\mathfrak{T}$ appears in Eq. (9) as an imaginary-time argument: According to Eq. (8), the solution $\psi_{\text {int }}^{-}(E)$ inside the barrier is determined by the Fourier transform of $e^{-\sqrt{2 m V_{0}} \chi(t)}$, evaluated at the energy shift $E-E_{\text {in }}$. This reflects the energy mixing at the front end $x=0$. For example, inserting an oscillating field $A(t)=A_{0} \sin (\omega t)$ which yields an oscillating $\chi(t)=$ $\chi_{0} \cos (\omega t)$, a Taylor expansion of the exponential $e^{-\sqrt{2 m V_{0}} \chi(t)}$ directly shows the appearance of side bands $E-E_{\text {in }}= \pm n \omega$.

Since modes with higher energy $E$ can tunnel easier though the barrier, they acquire an additional factor of $e^{\left(E-E_{\text {in }}\right) \mathfrak{T}}$ at the rear end of the barrier [see Eq. (6)]. Now we may calculate $\psi_{\text {tra }}(E)$ in Eq. (7) via the inverse Fourier transform of the wave function $\psi(t, x=L)$ at the rear end. After inserting Eqs. (6) and (8), we obtain two time integrals and one energy integration. Analytic continuation $t \rightarrow t+i \mathfrak{T}$ of the first time integral from Eq. (8) corresponding to the front end then allows us to cancel the additional factor of $e^{\left(E-E_{\text {in }}\right) \mathfrak{T}}$ such that the remaining energy integral just yields a Dirac delta distribution in time, giving the final result (9). Thus, this analytic continuation $t \rightarrow t+i \mathfrak{T}$ reflects the effect of energy mixing at the front end of the barrier.

\section{Dynamically assisted tunneling}

The result (9) now enables us to study the enhancement of tunneling due to the vector potential $A(t)$. In this form (9), we directly see the invariance under Galilei and gauge transformations $A(t) \rightarrow A(t)+$ const. Furthermore, it is interesting to note that the exponent $\sqrt{2 m V_{0}}[\chi(t+i \mathfrak{T})-\chi(t)]$ is consistent with the change of the instanton action [15]

$$
\sqrt{2 m V_{0}}[\chi(t+i \mathfrak{T})-\chi(t)]=-\sqrt{\frac{2 V_{0}}{m}} \int_{t}^{t+i \mathfrak{T}} d t^{\prime} q A\left(t^{\prime}\right),
$$

because $\sqrt{2 V_{0} / m}$ is the undisturbed instanton velocity. Thus, the above Eq. (11) is the analog to formula (10) in [16]. However, that work [16] was mainly focused on the tunneling exponent, whereas the above Eq. (9) does also contain the prefactor [for the box potential (4) and within the used approximations].

If we derive the typical energy gain or loss from Eq. (9) via the saddle-point method

$$
\begin{aligned}
\Delta E & =E-E_{\mathrm{in}} \approx-i \sqrt{2 m V_{0}}[\dot{\chi}(t+i \mathfrak{T})-\dot{\chi}(t)] \\
& =i q \sqrt{\frac{2 V_{0}}{m}}[A(t+i \mathfrak{T})-A(t)],
\end{aligned}
$$

we also find agreement with the instanton picture [15]. Note that the real time $t$ corresponds to the rear $x=L$ of the barrier, the complex time $t+i \mathfrak{T}$ to the front $x=0$ (see also [17]). 
As expected, the timescale distinguishing the adiabatic from the nonadiabatic (impulse) regime is the BüttikerLandauer traversal time (10). In the adiabatic regime of slowly varying $\chi(t)$, the difference $\chi(t+i \mathfrak{T})-\chi(t) \approx i \mathfrak{T} \dot{\chi}(t)$ is purely imaginary. Thus, the main effect is an energy shift $\Delta E$ given by $m L \ddot{\chi}(t)$, i.e., the energy gained in the electric field. A real contribution $\sqrt{2 m V_{0}} \boldsymbol{T}^{2} \ddot{\chi}(t) / 2$ to the exponent in Eq. (9) arises to second order in $\mathfrak{T}$. This contribution can be rewritten as $\mathfrak{T} \Delta E / 2$ and just reflects the quasistatic deformation of the potential by the electric field.

In the nonadiabatic regime of rapidly changing $\chi(t)$, however, we may get a stronger enhancement of the tunneling probability, as expected from the previous considerations. The first term $\chi(t+i \mathfrak{T})$ in the exponent in Eq. (9) stems from the front end $x_{\text {in }}^{E}=0$ of the barrier where the imaginary shift $t \rightarrow t+i \mathfrak{T}$ to complex time corresponds to energy mixing. The second term $\chi(t)$ stems from the rear end $x_{\text {out }}^{E}=L$ and reflects how the wave function is "pushed" out of the barrier. Altogether, we obtain the following rough estimate for the enhancement of the tunneling probability

$$
\mathcal{P}_{\chi} \sim \mathcal{P}_{0} \exp \left\{O\left(2 \sqrt{2 m V_{0}} \Delta \chi\right)\right\} .
$$

Note, however, that the simple result (9) has been derived for the box potential (4) and will not apply to other potential barriers $V(x)$ in general. The discontinuities at the front $x_{\text {in }}^{E}=0$ and the rear end $x_{\text {out }}^{E}=L$ make the energy mixing and displacement mechanisms quite efficient; this may change for other $V(x)$.

\section{TRIANGULAR POTENTIAL}

In order to study the difference between a very steep potential slope and a more gradual change, let us consider a triangular potential

$$
V(x)=\frac{x}{L} V_{0} \Theta(x) \Theta(L-x) .
$$

Of course, the ansatz for the solutions outside the barrier is the same as before in Eqs. (5) and (7). The solutions inside the barrier can be written in terms of displaced Airy functions $\mathrm{Ai}$ and $\mathrm{Bi}$ :

$$
\begin{aligned}
\psi(t, 0 & <x<L) \\
= & \int d E e^{-i E t+i \varphi(t)} \\
& \times\left\{\psi_{\text {int }}^{\mathrm{A}}(E) \mathrm{Ai}\left[\left(\frac{2 m V_{0}}{L}\right)^{1 / 3}\left(x-\chi(t)-\frac{E L}{V_{0}}\right)\right]\right. \\
& \left.+\psi_{\text {int }}^{\mathrm{B}}(E) \mathrm{Bi}\left[\left(\frac{2 m V_{0}}{L}\right)^{1 / 3}\left(x-\chi(t)-\frac{E L}{V_{0}}\right)\right]\right\},
\end{aligned}
$$

with the additional global phase $\dot{\varphi}(t)=V_{0} \chi(t) / L$ since an $x$ displacement of a linear potential $V(x) \propto x$ translates into a variation of the potential height.

As before, we apply the opaque-barrier and low-energy approximation $\chi(t) \ll L$ and $E \ll V_{0}$. In addition, the slope $V_{0} / L$ of the triangle is assumed to be small such that $V_{0} \chi(t) / L \ll E$. Under these assumptions, products of small terms are neglected (see Appendix A). Note that this includes the above phase $\varphi(t)$ as it scales with the product of the small slope $V_{0} / L$ and $\chi(t)$. However, as this phase $\varphi(t)$ does also include a time integral over $\chi(t)$, neglecting $\varphi(t)$ poses a restriction on the relevant timescales, which should not be too long. This is consistent with our previous considerations, as we are interested in rapid (i.e., nonadiabatic) changes.

Then, in analogy to the opaque-barrier approximation for the box potential, we find $\psi_{\text {int }}^{\mathrm{A}}(E) \ggg \psi_{\text {int }}^{\mathrm{B}}(E)$. Using the approximations described above, we find the transmitted solution

$$
\psi_{\mathrm{tra}}(E) \approx \psi_{E}^{0} \int \frac{d t}{2 \pi} e^{i\left(E-E_{\mathrm{in}}\right) t+\sqrt{2 m V_{0}} \chi(t)}
$$

where $\psi_{E}^{0}$ now describes the undisturbed $(\chi=0)$ amplitude for the triangle (14).

Comparing Eqs. (9) and (16), we find that the term $\chi(t+$ $i \mathfrak{T})$ describing the energy mixing at the front end is missing, just the "pushing out" effect at the rear end $x=L$ of the barrier remains. The suppression of energy mixing at the front end $x_{E}^{\text {in }}=E L / V_{0}$ of the barrier (i.e., the first classical turning point) can intuitively be understood by the nearly adiabatic evolution in space and time induced by the gradual change (with a very small slope).

\section{Quantum ratchets}

To complete the picture, let us consider the case of a triangular potential turned around, where the steplike discontinuity is at the front end while we have the gradual change at the rear end. As one might have expected from the previous considerations, we now obtain "the other half" of Eq. (9), i.e., the term $\chi(t+i \mathfrak{T})$ describing the mixing of energies at the front end, while the "pushing out" effect at the rear end has negligible effect due to the nearly adiabatic evolution in space and time induced by the gradual change.

Depending on the temporal structure of $\chi(t)$, the energy mixing $\chi(t+i \mathfrak{T})$ and the "pushing out" contribution $\chi(t)$ can be very different. For example, enhancing the tunneling probability by "pushing out" part of the wave function obviously requires $\chi>0$, while the energy mixing contribution $\chi(t+$ $i \mathfrak{T})$ can also enhance tunneling if the electric field points in the other direction, provided that its temporal structure (e.g., spectrum) contains large enough frequency components.

In stationary tunneling, one cannot observe such a difference between the triangular potential (14) and its mirror image. Due to unitarity, the tunneling probability does not depend on whether the wave is incident from left or right. The time-dependent vector potential $A(t)$, however, induces a nonequilibrium situation, where such a breaking of symmetry is possible. This phenomenon is closely related to quantum ratchets (see, e.g., [18,19]).

\section{EXPERIMENTAL REALIZATIONS}

Since tunneling plays a role in various areas of physics, its dynamical assistance could be observed in several scenarios. However, as most of them will not correspond to a rectangular or shallow triangular potential, let us first briefly discuss the case of a general potential $V(x)$. 


\section{A. Büttiker-Landauer traversal time}

For general potentials $V(x)$, the Büttiker-Landauer traversal time $\mathfrak{T}$ is given by [14]

$$
\mathfrak{T}=-\frac{d \mathfrak{S}_{E}}{d E}=\int_{x_{\mathrm{in}}^{E}}^{x_{\mathrm{out}}^{E}} d x \sqrt{\frac{m}{2[V(x)-E]}} .
$$

This quantity plays a manifold role. It describes the (imaginary) propagation time of an instanton from $x_{\text {in }}^{E}$ to $x_{\text {out }}^{E}$. Furthermore, it measures how much the instanton action $\mathfrak{S}_{E}$ (which determines the tunneling exponent) decreases when increasing the energy $E$. As a consequence, the BüttikerLandauer traversal time $\mathfrak{T}$ provides an estimate for the frequency components $\omega$ a pulse (or time-dependent field) should contain to facilitate a significant enhancement of tunneling. If the characteristic frequency components $\omega$ are too low $\omega \mathfrak{T} \ll 1$, the energy mixing is not sufficient to shift the instanton action $\mathfrak{S}_{E}$ enough. As a result, the Büttiker-Landauer traversal time $\mathfrak{T}$ can be used to separate slow (adiabatic) from fast (nonadiabatic) processes.

Note, however, that this quantity $\mathfrak{T}$ does not yield any information about the efficiency of the energy mixing or "pushing out" processes. For example, $\mathfrak{T}$ does not depend on whether the particle is incident from left or right (cf. Sec. IV). The Büttiker-Landauer traversal time $\mathfrak{T}$ corresponds to the change of the tunneling exponent, but does not describe the prefactor in front of the exponential. In the Floquet picture, for example, these prefactors are determined by the matrix elements between the Floquet bands, which determine the efficiency of the energy mixing or "pushing out" processes.

It should also be stressed here that the Büttiker-Landauer traversal time $\mathfrak{T}$ is not necessarily the unique answer to the question of how long the particle stays inside the barrier during tunneling; this is indeed a nontrivial issue (already at the stage of a proper definition, see also [20-22]). Instead, it is an important quantity for discriminating slow from fast processes, as explained above. In this role, it provides a good first estimate of the experimental requirements for observing assisted tunneling. From Eq. (17), we may read off the rough scaling law $\mathfrak{T}=O(L \sqrt{m /[V-E]})$ where $L$ is again the length of the barrier. In analogy, the instanton action $\mathfrak{S}_{E}$ in Eq. (1) can be estimated via $\mathfrak{S}_{E}=O(L \sqrt{m[V-E]})$. Now, since $\mathfrak{S}_{E}$ should not be too large in order to have a measurable tunneling probability, we obtain the rough orderof-magnitude estimate $\mathfrak{T} \sim O\left(L^{2} m\right)$. This behavior of the characteristic timescale can also be obtained directly from the Schrödinger equation by comparing the temporal derivative term $i \partial_{t} \psi$ with the kinetic energy contribution $\nabla^{2} \psi /(2 m)$.

\section{B. Ultracold atoms in optical lattices}

On a comparably large length scale of order micrometer, optical lattices generated by standing laser beams in the optical or near-optical regime provide a potential landscape for ultracold atoms in which they can tunnel from one potential minimum to the next one (see, e.g., [2,3]). The potential barrier height can be tuned by the laser strength (and its detuning). The above estimate $\mathfrak{T} \sim O\left(L^{2} m\right)$ of the BüttikerLandauer traversal time scales as the inverse of the recoil energy $E_{\mathrm{R}}=k^{2} /(2 m)$ which is typically of the order of tens of $\mathrm{kHz}$. Nonadiabatic variations should then be in the submillisecond regime, which is not beyond the experimental capabilities. An effective electric field can be generated by accelerated motion of the optical lattice (i.e., a real displacement in space), which can be understood as an inverse of the Kramers-Henneberger transformation. This realization allows us to study dynamically assisted tunneling for neutral particles such as atoms [23-27].

\section{Electrons in solids}

Another prototypical example are electrons in solid-state devices with characteristic length scales between the nanometer and the micrometer scale [28-33]. By fabricating these devices and applying gate voltages, one could even appropriately realize a box (4) or triangular (14) potential. Due to the smaller length scales and the smaller (effective) mass of the electron, the Büttiker-Landauer traversal time is much shorter in this case and corresponds to frequencies ranging from the terahertz to the infrared regime (see, e.g., [34]), which can be coupled in via real electromagnetic fields.

\section{Atomic physics}

On even smaller length scales in the nanometer or subnanometer regime, electrons tunnel from one atomic or molecular orbital to another or into free space (field ionization) [35-37]. The reduction of the characteristic length scales goes along with a further increase of the typical frequency scales necessary to reach the nonadiabatic regime [38-40], which range from the optical or near-optical frequencies (see, e.g., $[41,42])$ up to the keV regime for tightly bound electrons around highly charged (high-Z) nuclei (cf. [43]).

\section{E. Nuclear $\alpha$ decay}

Tunneling on yet smaller length scales in the picometer to femtometer regime plays an important role in nuclear physics. As one of the first applications of quantum tunneling, Gamov explained the Geiger-Nuttall law of nuclear $\alpha$ decay [5] via tunneling of the $\alpha$ particle through the Coulomb barrier of the remaining nucleus [4]. Of course, it would be interesting to study the option for dynamically assisting this process, for example, with the strong field generated by an x-ray freeelectron laser (XFEL). This topic has induced several, partly controversial, discussions in recent years (see, e.g., [44,45]).

Following our strategy above, let us estimate the BüttikerLandauer traversal time for this case. As shown in [46], for example, the associated frequency scales are in the $100 \mathrm{keV}$ to $\mathrm{MeV}$ regime (see also $[45,47]$ ). Even though the mass of the $\alpha$ particle is much larger than the electron mass, the extremely small length scales lead to ultrashort times. Since frequencies in the $100 \mathrm{keV}$ to $\mathrm{MeV}$ regime are probably hard to reach with current or near-future XFEL facilities, one should search for alternatives. One such option for creating short enough pulses could be the electromagnetic field generated by an additional nucleus (see also [48]) with an energy of order $50 \mathrm{MeV}$ passing by at a distance of order 100 femtometers. Of course, this electromagnetic field is not really spatially homogeneous, but the main effects should persist, at least qualitatively. 


\section{NUCLEAR FUSION}

In the following, let us study nuclear fusion in more detail (see, e.g., [16,49-54]), which can be regarded as the process opposite to nuclear $\alpha$ decay (or, more general, nuclear fission). Interesting examples include deuterium-tritium (see, e.g., $[53,54])$

$$
{ }_{1}^{2} \mathrm{D}+{ }_{1}^{3} \mathrm{~T} \rightarrow{ }_{2}^{4} \mathrm{He}+{ }_{0}^{1} n+17.6 \mathrm{MeV},
$$

or proton-boron fusion (see, e.g., [55-58])

$$
{ }_{1}^{1} p+{ }_{5}^{11} \mathrm{~B} \rightarrow 3 \times{ }_{2}^{4} \mathrm{He}+8.7 \mathrm{MeV} .
$$

First, we focus on the most simple case of two particles with masses $m_{1}$ and $m_{2}$ and charges $q_{1}$ and $q_{2}$ in the initial state. Later we shall discuss the generalization to more complicated scenarios such as muon-assisted fusion.

\section{A. The model}

Describing the two nuclei as nonrelativistic point particles (in the low-energy regime), their dynamics is governed by the two-body Lagrangian

$$
\begin{aligned}
L_{12}= & \frac{m_{1}}{2} \dot{\boldsymbol{r}}_{1}^{2}+\frac{m_{2}}{2} \dot{\boldsymbol{r}}_{2}^{2}-V\left(\left|\boldsymbol{r}_{1}-\boldsymbol{r}_{2}\right|\right) \\
& +\left(q_{1} \dot{\boldsymbol{r}}_{1}+q_{2} \dot{\boldsymbol{r}}_{2}\right) \cdot \boldsymbol{A}(t),
\end{aligned}
$$

where the potential $V\left(\left|\boldsymbol{r}_{1}-\boldsymbol{r}_{2}\right|\right)$ contains the Coulomb repulsion at large distances and the nuclear attraction at short distances. The vector potential $\boldsymbol{A}$ represents the field of the XFEL, which can be approximated by a purely timedependent field because the XFEL wavelength is much larger than the characteristic length scales of our problem (see also [16]).

In center of mass $\boldsymbol{R}=\left(m_{1} \boldsymbol{r}_{1}+m_{2} \boldsymbol{r}_{2}\right) /\left(m_{1}+m_{2}\right)$ and relative coordinates $\boldsymbol{r}=\boldsymbol{r}_{1}-\boldsymbol{r}_{2}$, the effective single-body Lagrangian for the latter reads as

$$
L=\frac{m}{2} \dot{\boldsymbol{r}}^{2}-V(|\boldsymbol{r}|)+q_{\mathrm{eff}} \dot{\boldsymbol{r}} \cdot \boldsymbol{A}(t),
$$

with the reduced mass $1 / m=1 / m_{1}+1 / m_{2}$ and the effective charge $q_{\text {eff }}=\left(q_{1} m_{2}-q_{2} m_{1}\right) /\left(m_{1}+m_{2}\right)$. Upon quantization, we arrive at the same Schrödinger equation (2), but with rescaled variables $m$ and $q_{\text {eff }}$.

\section{B. Scaling analysis}

In order to identify the relevant parameters, let us first perform a scaling analysis of the Schrödinger equation (2) which is facilitated by the self-similarity of the Coulomb potential. Neglecting the details of the nuclear attraction at short distances (in a low-energy approximation), the potential $V(|\boldsymbol{r}|)$ is a homogeneous function of degree -1 , i.e., $V(|\lambda \boldsymbol{r}|)=$ $V(|\boldsymbol{r}|) / \lambda$. This allows us to cast the Schrödinger equation (2) into a dimensionless form. Using the initial energy $E$ in order to set the frequency and timescale, the length scale can be set by the outer (classical) turning point $r_{E}$ where $V\left(r_{E}\right)=E$. Then the comparison of the kinetic term $\nabla^{2} \psi /(2 m)$ with the energy $E \psi$ yields the first dimensionless parameter

$$
\eta=2 m E r_{E}^{2}=\frac{2 m}{E}\left(\frac{q_{1} q_{2}}{4 \pi \epsilon_{0}}\right)^{2} .
$$

The square root of this parameter yields the undisturbed WKB tunneling exponent $\mathcal{P} \sim \exp \{-\pi \sqrt{\eta}\}$ in Eq. (1) and the Büttiker-Landauer traversal time $E \mathfrak{T}=\pi \sqrt{\eta} / 4$ for this potential. As explained above, this WKB tunneling exponent (and thus $\eta$ ) should not become too large in order to have a measurable tunneling probability.

The second dimensionless parameter can be constructed by incorporating the remaining term $q_{\text {eff }} \boldsymbol{A}$ in the Schrödinger equation (2). Of course, in view of the dimensionless parameters (22) and $E / \omega$, this construction is not unique. Motivated by the above considerations, we choose to compare the amplitude of the displacement (3) with $r_{E}$ giving

$$
\zeta=\frac{q_{\mathrm{eff}} A}{m \omega r_{E}}=\frac{q_{\mathrm{eff}} A}{m c} \frac{E}{\omega} \frac{4 \pi \epsilon_{0} c}{q_{1} q_{2}},
$$

where the last ratio on the right-hand side is the inverse of the QED fine-structure constant modified by the charge numbers $Z_{1}$ and $Z_{2}$ of the two nuclei. The first ratio is analogous to the inverse Keldysh parameter $1 / \gamma$ or the laser parameter $a_{0}$, but now with the electron mass being replaced by the reduced mass of the nuclei. Hence, this quantity $\zeta$ will typically be smaller than unity, but again it should not be too small to see a significant effect.

The fact that the two dimensionless parameters $\eta$ and $\zeta$ in Eqs. (22) and (23) should not be too far above or below unity, respectively, shows that we do not have good scale separation in our problem, which makes it hard to distinguish the four effects (preacceleration, energy mixing, potential deformation, and displacement) mentioned in the Introduction. Instead, they will all be intertwined. Still, the previous results, especially the comparison between the rectangular and the triangular potentials, suggest that energy mixing may be less efficient than displacement, for example, due to the gradual change of the Coulomb potential at the outer turning point $r_{E}$.

As another lesson, we may compare deuterium-tritium with proton-boron fusion by means of the above scaling analysis. For the proton-boron system, the Coulomb strength $\propto q_{1} q_{2}$ is a factor of 5 stronger than in the deuterium-tritium case, while the reduced mass is roughly a factor of $\frac{3}{4}$ smaller. According to Eq. (22), the energy should thus be nearly a factor of 20 larger in order to achieve the same WKB tunneling probability. Note, however, that this rough estimate is based on neglecting the details of the nuclear attraction, i.e., the real factor will be a bit smaller than 20 . Nevertheless, as the effective charge $q_{\text {eff }}$ of the proton-boron system is approximately a factor of $\frac{5}{2}$ larger than in the deuterium-tritium case, the required vector potentials do not differ much (only by a factor of $\frac{3}{2}$ ) in the two cases.

\section{Numerical results}

As explained above, the analytical scaling analysis in the previous section does not take into account the fact that the Coulomb potential is cut off at nuclear distances of a few femtometers. In order to include this effect and to arrive at quantitative results, we solved the onedimensional Schrödinger equation in the presence of the cutoff Coulomb potential $V(x)$ and the time-dependent pulse $A_{x}(t)=A_{0} / \cosh ^{2}(\omega t)$ numerically for initial Gaussian wave packets (see Appendix B). 
Let us first discuss the case of deuterium-tritium fusion with an effective charge $q_{\text {eff }} \approx q / 5$ and reduced mass $m \approx$ $1 \mathrm{GeV}$. For initial kinetic energies of 2,4 , and $8 \mathrm{keV}$, the outer classical turning points $r_{E}$ determined by the Coulomb repulsion, which correspond to the initial turning points $x_{\text {in }}^{E}$ in Eq. (1), are $r_{E} \approx 720,360$, and $180 \mathrm{fm}$, respectively. The inner turning point, corresponding to $x_{\text {out }}^{E}$ in Eq. (1), is determined by the nuclear attraction and lies around $4 \mathrm{fm}$.

For these energies $(2,4$, and $8 \mathrm{keV})$, the Büttiker-Landauer traversal times are given by $\mathfrak{T} \approx 2,0.7$, and 0.25 as, respectively, while the dimensionless parameters in Eq. (22) assume the values $\eta \approx 60,30$, and 15 , respectively. In view of $\hbar \approx$ $0.7 \mathrm{keV}$ as, we see that we may probe nonadiabatic effects with frequencies in the $\mathrm{keV}$ regime.

A pulse $A_{x}(t)$ with $\omega=1 \mathrm{keV}$ and a peak field strength of $10^{16} \mathrm{~V} / \mathrm{m}$ then corresponds to a Kramers-Henneberger displacement of $\Delta \chi \approx 130 \mathrm{fm}$. Hence, the dimensionless parameter in Eq. (23) assumes the values $\zeta \approx 0.1,0.2$, and 0.4 for these energies $(2,4$, and $8 \mathrm{keV})$. Note, however, that the above value of $\Delta \chi \approx 130 \mathrm{fm}$ does not necessarily correspond to a real displacement of the wave function (this would only be the case in the sudden approximation). As we can see in Fig. 1, for example, the impact of the field on the dynamics does also depend on the other parameters involved.

The enhancement of the tunneling rate [defined as the probability current $j(t)$ on the rear end of the barrier] is displayed in Fig. 1. For all three values of the initial kinetic energy $(2,4$, and $8 \mathrm{keV})$, we see that we obtain a significant enhancement whose relative strength decreases a bit with increasing energy. As explained above, the absence of good scale separation makes it hard to disentangle the four contributions (preacceleration, energy mixing, potential deformation, and displacement). However, comparison with the results of Ref. [16] where mainly the tunneling exponent has been considered [see Eq. (10) in that work, which is the analog of our formula (11) for the box potential] suggests that the energy mixing contribution is suppressed by the gradual change of the Coulomb potential near the outer turning point $r_{E}$, which is consistent with our results for the triangular potential.

As motivated by the scaling analysis above, let us compare these results for deuterium-tritium fusion with the protonboron scenario. First, in order to obtain approximately the same dimensionless parameters $\eta$ and $\zeta$ as in the deuteriumtritium case with $E=2 \mathrm{keV}$, we choose an initial kinetic energy of $E=38 \mathrm{keV}$ and a pulse with $\omega=19 \mathrm{keV}$ and $28 \times 10^{16} \mathrm{~V} / \mathrm{m}$. The outer classical turning point is then $r_{E} \approx$ $190 \mathrm{fm}$ and the Büttiker-Landauer traversal time $\mathfrak{T} \approx 0.1$ as. Comparing the top plots in Figs. 1 and 2, we indeed find rather good agreement of the relative enhancement rates, even though the inner turning point (a bit above $4 \mathrm{fm}$ for the protonboron case) does not follow the scaling transformation.

For comparison, we also considered the impact of the same pulse (with $\omega=1 \mathrm{keV}$ and $10^{16} \mathrm{~V} / \mathrm{m}$ ) as in the deuteriumtritium case. As one may observe in Fig. 2 (middle and bottom), such a pulse does also yield significant enhancement rates for initial kinetic energies of 40 and $80 \mathrm{keV}$. However, in view of the shorter Büttiker-Landauer traversal times $\mathfrak{T} \approx 0.1$ and 0.03 as, such a pulse with $\omega=1 \mathrm{keV}$ may already be too slow to probe nonadiabatic effects.
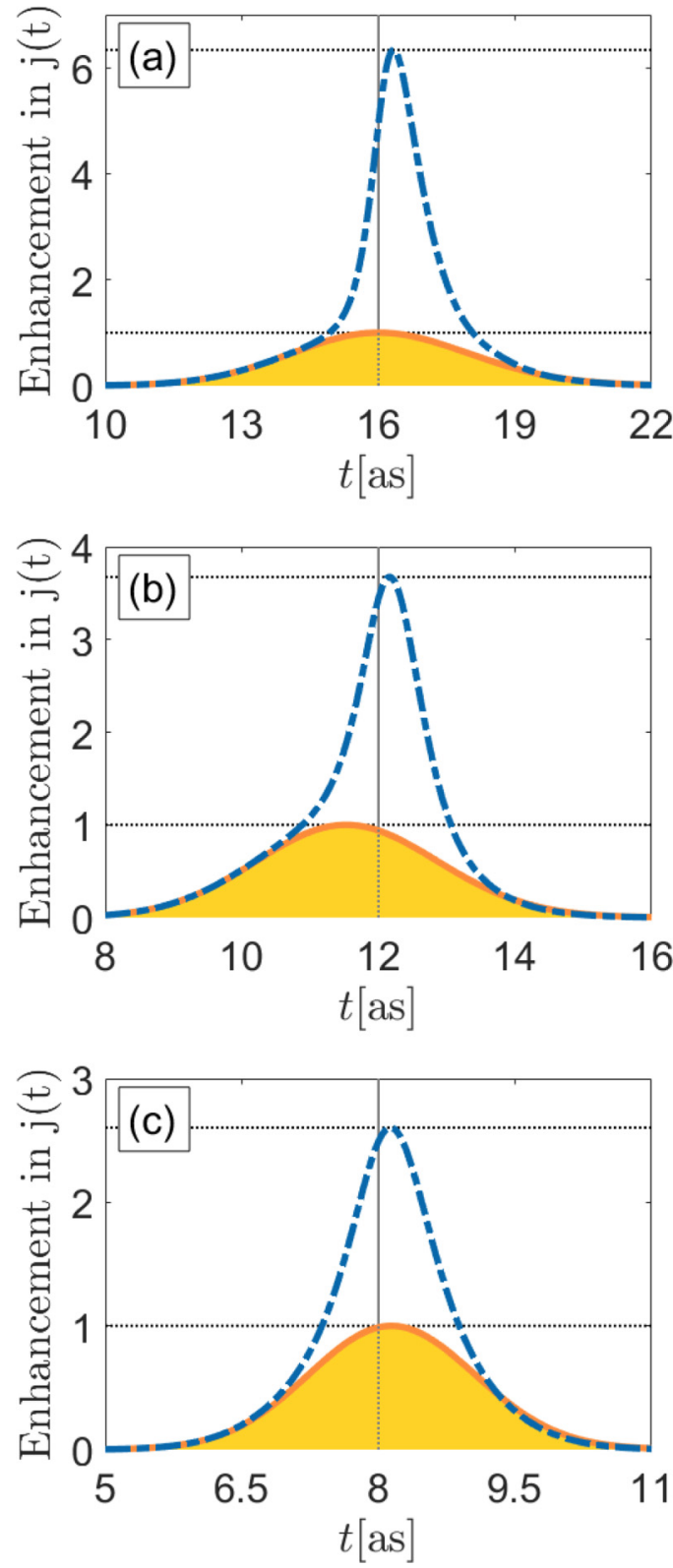

FIG. 1. Enhancement of the tunneling rate for deuterium-tritium fusion with initial kinetic energies of 2 (top), 4 (middle), and 8 $\mathrm{keV}$ (bottom). The orange curves enclosing the yellow bell-shaped regions correspond to the undisturbed tunneling rates $j(t)$ of the initial Gaussian wave packets without the electric field while the blue dashed-dotted curves show the enhancement due to the pulse $A_{x}(t)$ with $\omega=1 \mathrm{keV}$ and a peak field strength of $10^{16} \mathrm{~V} / \mathrm{m}$.

\section{CONCLUSIONS}

We study how tunneling of a charged particle through a static potential barrier $V(x)$ could be dynamically assisted by an additional time-dependent electric field. We identify four main mechanisms corresponding to the stages of the temporal evolution or the spatial regions: (i) preacceleration before the barrier, (ii) energy mixing at its front end, (iii) deformation of the potential barrier, and (iv) displacement at its rear end. While the two effects (i) and (iii) are already present in the adiabatic regime of slowly varying electric fields, the other 

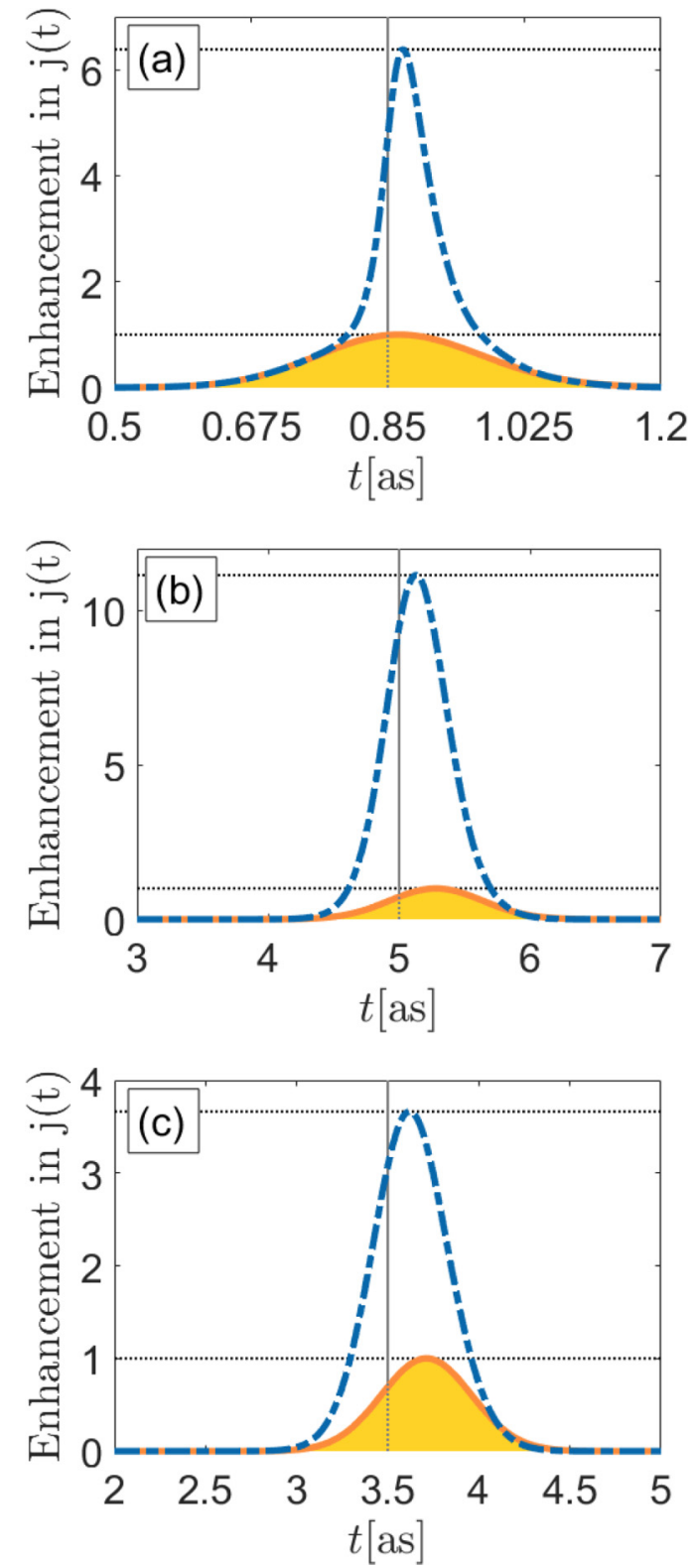

FIG. 2. Enhancement of the tunneling rate for proton-boron fusion. The top plot corresponds to an initial kinetic energy of $38 \mathrm{keV}$ and a pulse with $\omega=19 \mathrm{keV}$ and $28 \times 10^{16} \mathrm{~V} / \mathrm{m}$, which represents the scaling transformation of the scenario in Fig. 1 (top). For comparison, using the same pulse with $\omega=1 \mathrm{keV}$ and $10^{16} \mathrm{~V} / \mathrm{m}$ as in Fig. 1 for deuterium-tritium fusion does also yield significant enhancement for initial kinetic energies of 40 (middle) and $80 \mathrm{keV}$ (bottom).

two phenomena (ii) and (iv) require sufficiently fast, i.e., nonadiabatic, changes of the electric field.

For the special cases of rectangular and triangular potential barriers, we we were able to disentangle the four contributions by means of approximate analytic solutions. We found that the two nonadiabatic effects (ii) energy mixing and (iv) displacement (occurring at the front and rear end, respectively) are dual to each other with the main difference that the former one (ii) is associated to an analytic continuation to complex times $t \rightarrow t+i \mathfrak{T}$ where $\mathfrak{T}$ is the Büttiker-Landauer traversal time.
TABLE I. Rough order-of-magnitude estimate of the characteristic length and energy scales for various tunneling scenarios and the typical field strengths required for dynamical assistance.

\begin{tabular}{lccc}
\hline \hline Length & System & Energy & Field strength \\
\hline$\mu \mathrm{m}$ & Optical lattices & $\mathrm{peV}$ & $\mathrm{n.a.}$ \\
& Solids & $\mathrm{meV}$ & $10^{5} \mathrm{~V} / \mathrm{m}$ \\
$\mathrm{nm}$ & Atoms & $\mathrm{eV}$ & $10^{10} \mathrm{~V} / \mathrm{m}$ \\
& Nuclear fusion & $\mathrm{keV}$ & $10^{16} \mathrm{~V} / \mathrm{m}$ \\
$\mathrm{pm}$ & $\alpha$ decay & & $10^{18} \mathrm{~V} / \mathrm{m}$ \\
$\mathrm{fm}$ & & $\mathrm{MeV}$ & \\
\hline \hline
\end{tabular}

This analytic continuation reflects the energy shift, which in turn changes the tunneling through the barrier.

The occurrence of a complex time is also related to another distinction between the two contributions (ii) and (iv). The displacement effect (iv) strongly depends on the sign of the electric field: In one direction, the electric field would "push" parts of the wave function out of the rear end of the barrier and thereby enhance tunneling, while an electric field pointing in the opposite direction would "pull" them into the barrier again and thereby suppress tunneling. In contrast, the energy mixing effect (ii) can enhance tunneling for both signs of the electric field, especially deep in the nonadiabatic regime, where the shift of the energy induced by the time dependence of the electric field becomes the most important contribution (see also Appendix C).

Furthermore, the comparison between the rectangular and the triangular potentials indicates that the efficiency of the two nonadiabatic effects (ii) and (iv) strongly depends on the shape of the potential at the turning points and is suppressed in the case of a gradual change. This finding is related to the quantum ratchet effect where tunneling in one direction may be favored compared to the other in nonequilibrium situations.

The Büttiker-Landauer traversal time $\mathfrak{T}$ mentioned above is an important quantity in this respect because it can be used to distinguish slow (i.e., adiabatic) from fast (i.e., nonadiabatic) processes. Since tunneling is a crucial effect in many areas of physics, we briefly discuss the observability of the considered mechanisms in several scenarios, such as ultracold atoms in optical lattices, electrons in solids and in atoms and molecules, and nuclear $\alpha$ decay (see also Table I).

Finally, we turn our attention to nuclear fusion and discuss the special scaling properties of this process stemming from the Coulomb potential. As an explicit example, we study a Sauter pulse $A_{x}(t)=A_{0} / \cosh ^{2}(\omega t)$ with $\omega=1 \mathrm{keV}$ and a peak field strength of $10^{16} \mathrm{~V} / \mathrm{m}$ and find a significant enhancement of the tunneling rate for deuterium-tritium fusion with initial energies between 2 and $8 \mathrm{keV}$ as well as for proton-boron fusion in the $40-80 \mathrm{keV}$ energy range.

Of course, the required field strength of $10^{16} \mathrm{~V} / \mathrm{m}$ is quite large, but still well below the Schwinger critical field $E_{S}=$ $m_{e}^{2} c^{3} /(q \hbar) \approx 1.3 \times 10^{18} \mathrm{~V} / \mathrm{m}$. Even though it is probably beyond the present capabilities of $\mathrm{x}$-ray free-electron lasers (XFEL), such field strengths might be achievable with further technological progress, e.g., focusing the XFEL beam better. Another interesting option could be high-harmonic focusing [59-61] or similar effects of light-matter interaction at 
ultrahigh intensities (cf. [62]). As a different approach, such ultrashort and ultrastrong pulses (though with a potentially non-negligible spatial dependence) could be generated by charged particles (such as $\alpha$ particles) with sufficiently high energies (e.g., $50 \mathrm{keV}$ or above) and small enough impact parameters (e.g., $500 \mathrm{fm}$ or below). Note that highly energetic $\alpha$ particles are actually produced in deuterium-tritium and, even more so, in proton-boron fusion, such that this enhancement process might perhaps be (partially) self-sustained after an initial ignition phase. While the additional spatial dependence of the Coulomb field of these $\alpha$ particles complicates the analysis [63], the main effects such as the displacement mechanism should persist, at least qualitatively.

\section{OUTLOOK}

As became evident from the previous considerations, our understanding is by far not complete yet and there are many ways for further progress. For example, one could generalize the approximate analytical solutions derived above to other potentials, such as combinations of piecewise constant, linear, or even parabolic potential barriers. Another obvious generalization is the solution of the full three-dimensional (3D) Schrödinger equation, e.g., in position representation or after an expansion into spherical harmonics. Even though one would expect the main mechanisms (such as the displacement) to persist, there should be quantitative differences, e.g., regarding the prefactors.

This could also pave the way to study the impact of electromagnetic fields $\boldsymbol{A}(t, \boldsymbol{r})$ depending on space and time. Related studies of the Sauter-Schwinger effect (see, e.g., $[46,64,65]$ ) show a nontrivial interplay between the spatial and the temporal dependence of the field. In this respect, it could be interesting to compare the assisted tunneling studied in this work with the dynamically assisted Sauter-Schwinger effect [66] which is governed by a relativistic (Dirac or Klein-FockGordon) wave equation instead of the Schrödinger equation.

\section{Muon-assisted fusion}

As one can observe in Fig. 1, the relative enhancement of the tunneling rate does, in stark contrast to the tunneling rate itself, not depend very strongly on the initial kinetic energy, which is consistent with the expected behavior of the displacement mechanism, for example. Thus, instead of starting with an asymptotically free scattering state (which was the scenario studied above), one could consider an initial bound state created by a dip in the Coulomb potential. This could be regarded as a toy model for muon-assisted nuclear fusion where the deuterium and tritium nuclei form a bound state with a muon (see, e.g., [67-70]).

Motivated by this toy model, we solved the Schrödinger equation in the presence of the same pulse $A_{x}(t)$ with $\omega=$ $1 \mathrm{keV}$ and $10^{16} \mathrm{~V} / \mathrm{m}$ as before, but now with a potential $V(x)$ admitting a bound state, which we used as our initial state (see Appendix B 4). The result is plotted in Fig. 3 and shows that such a pulse can also induce a significant enhancement in this case. Even though the potential $V(x)$ is a bit different, one would expect that the displacement mechanism operates in a very similar way, as the vicinity of the inner turning

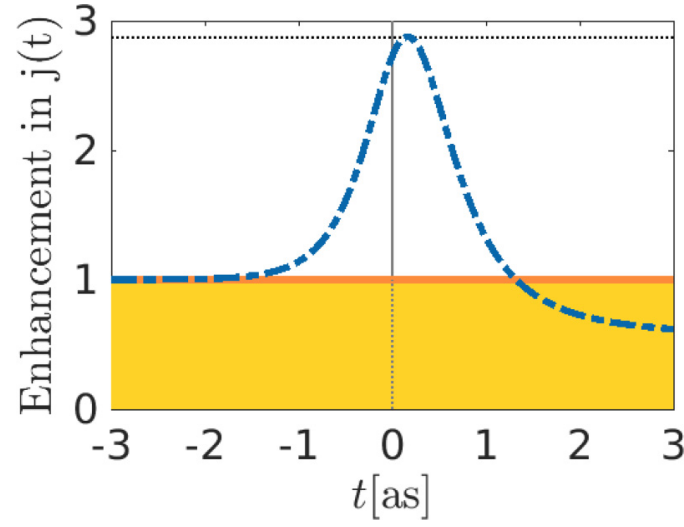

FIG. 3. Enhancement of the tunneling rate starting in a bound state as a toy model for muon-assisted deuterium-tritium fusion, again a pulse with $\omega=1 \mathrm{keV}$ and $10^{16} \mathrm{~V} / \mathrm{m}$ has been assumed.

point is basically unchanged. However, the preacceleration mechanism should be strongly affected as it acts on the initial state.

Furthermore, it should be stressed here that the case of muon-assisted fusion is much more complex. For example, the electric field does not only couple to the relative motion of the deuterium and tritium nuclei via $q_{\text {eff }}$, it also couples directly to the oppositely charged muon (which is much lighter). Thus, we have a real three-body problem here [71], which is far more involved, but also offers far more interesting possibilities. This includes the eigenfrequencies of the three-body problem which partially also lie in the $\mathrm{keV}$ regime [72] and thus facilitate a strong (and possibly resonant) coupling to the external electric field. For a bigger picture, one should reconsider the whole process in the presence of the external electromagnetic (e.g., XFEL) field, including the unwanted sticking of the muon to the produced $\alpha$ particles, which might also be affected by the external field.

\section{ACKNOWLEDGMENTS}

The authors thank N. Ahmadiniaz, R. Sauerbrey, G. Torgrimsson, and other colleagues from the HZDR for fruitful discussions. This work was funded by the Deutsche Forschungsgemeinschaft (DFG, German Research Foundation), Projects No. 398912239 and No. 278162697-SFB 1242.

\section{APPENDIX A: TRIANGULAR POTENTIAL}

For the analytic treatment of the triangular potential in Sec. IV, we employed the low-energy and opaque-barrier approximation as well as the assumption of a small slope. In order to demonstrate the consistency of these three limits and how they can be based on a systematic expansion, we introduce a small parameter $\varepsilon$ and consider the limit $\varepsilon \downarrow 0$. The low-energy approximation is then recovered by the scaling of the involved energies

$$
E \rightarrow \sqrt{\varepsilon} E,
$$

and analogously $E_{\text {in }} \rightarrow \sqrt{\varepsilon} E_{\text {in }}$ while the potential barrier height $V_{0}$ stays constant, i.e., independent of $\varepsilon$. 
Given that the barrier height $V_{0}$ stays constant, we scale the barrier length as

$$
L \rightarrow \frac{L}{\varepsilon}
$$

which corresponds to the opaque-barrier approximation, and, at the same time, to the assumption of a small slope. For convenience, we also scale the time $t$ as $t \rightarrow t / \sqrt{\varepsilon}$ such that the $\sqrt{\varepsilon}$ factors in the product $E t$ cancel each other, and similarly the wave functions $\psi \rightarrow \psi / \sqrt{\varepsilon}$.

In this limit $\varepsilon \downarrow 0$, the tunneling exponent scales as $1 / \varepsilon$ which reflects the nonperturbative character of tunneling. In comparison, the corrections induced by the time-dependent perturbation $\chi=O\left(\varepsilon^{0}\right)$ are small because $\chi$ is smaller than the barrier length $\propto 1 / \varepsilon$ as well as the turning point $x_{E}^{\text {in }} \propto$ $1 / \sqrt{\varepsilon}$.

\section{Gradual incidence}

Let us first consider a wave incident on the triangular potential (14) from the left-hand side. The solutions inside the barrier read as

$$
\psi(t, 0<x<L / \varepsilon)=\int d E e^{-i E t+i \varepsilon \varphi} \times\left\{\psi_{\text {int }}^{\mathrm{A}}(E) \operatorname{Ai}[g(x-\chi)]+\psi_{\text {int }}^{\mathrm{B}}(E) \operatorname{Bi}[g(x-\chi)]\right\},
$$

where we have used the abbreviation

$$
g(x)=\left(\frac{2 \varepsilon m V_{0}}{L}\right)^{1 / 3}\left(x-\frac{E L}{\sqrt{\varepsilon} V_{0}}\right) .
$$

In the following, we neglect the global phase $\varphi$ as it scales with $\varepsilon$.

Let us consider the junction conditions at the front end $x=0$ of the barrier. Because the Airy function Ai is exponentially decreasing for large positive arguments whereas the other $\mathrm{Bi}$ is exponentially increasing, the amplitudes of the interior solution satisfy $\psi_{\mathrm{int}}^{\mathrm{A}} \ggg \psi_{\mathrm{int}}^{\mathrm{B}}$ as the tunneling wave function will be exponentially suppressed at the rear end of the barrier. Since both Airy functions around $x=0$ are of order unity, we can neglect the coefficients $\psi_{\text {int }}^{\mathrm{B}}(E)$ in the junction conditions at $x=0$. We find to leading order

$$
e^{-i E_{\mathrm{in}} t}+\int d E \psi_{\mathrm{refl}}(E) e^{-i E t}=\int d E \psi_{\mathrm{int}}^{\mathrm{A}}(E) \mathrm{Ai}\left[-z_{E}\right] e^{-i E t}
$$

and

$$
i \sqrt{2 m \sqrt{\varepsilon} E_{\mathrm{in}}} e^{-i E_{\mathrm{in}} t}-\int d E i \sqrt{2 m \sqrt{\varepsilon} E} \psi_{\mathrm{refl}}(E) e^{-i E t}=\int d E \psi_{\mathrm{int}}^{\mathrm{A}}(E)\left(\frac{2 \varepsilon m V_{0}}{L}\right)^{1 / 3} \mathrm{Ai}^{\prime}\left[-z_{E}\right] e^{-i E t}
$$

with the abbreviation

$$
z_{E}=\left(\frac{2 \varepsilon m V_{0}}{L}\right)^{1 / 3} \frac{E L}{\sqrt{\varepsilon} V_{0}}=-g(0)
$$

Solving Eqs. (A5) and (A6) via a Fourier transformation, the dominant contribution reads as

$$
\psi_{\text {int }}^{A}(E)=2 \sqrt{\pi} \delta\left(E-E_{\text {in }}\right) z_{E}^{1 / 4} \exp \left\{i\left(\frac{2}{3} z_{E}^{3 / 2}-\frac{\pi}{4}\right)\right\},
$$

where we have used the asymptotic expansion of the Airy function for large negative arguments since $z_{E} \propto \varepsilon^{-1 / 6}$ due to the the smallness of the potential slope. We see immediately from (A8) that no energy mixing occurs when the wave function enters the gradually increasing barrier.

Let us turn to the junction conditions at the rear end $x=L / \varepsilon$. Here we cannot neglect the exponentially small functions $\psi_{\text {int }}^{\mathrm{B}}$ since they are multiplied with an exponentially large factor. In view of the large tunneling exponent $\propto 1 / \varepsilon$, we employ the asymptotic expressions of the Airy functions for large positive arguments. Altogether, we obtain for the transmitted amplitude

$$
\begin{aligned}
\psi_{\text {trans }}(E)= & \exp \left\{-i \sqrt{2 m E \sqrt{\varepsilon}} \frac{L}{\varepsilon}+i\left(\frac{2}{3} z_{E_{\text {in }}}^{3 / 2}-\frac{\pi}{4}\right)\right\} \\
& \times 2\left(\frac{\sqrt{\varepsilon} E_{\text {in }}}{V_{0}}\right)^{\frac{1}{4}} \int \frac{d t}{2 \pi} e^{i\left(E-E_{\text {in }}\right) t} \times \exp \left\{-\frac{2}{3}\left(2 m V_{0}\right)^{1 / 2} \frac{L}{\varepsilon}+\frac{E_{\text {in }} \mathfrak{T}}{\sqrt{\varepsilon}}+\left(2 m V_{0}\right)^{1 / 2} \chi\right\} .
\end{aligned}
$$

In the exponential in the last line, the dominant contribution $\propto 1 / \varepsilon$ is just the undisturbed tunneling exponent at zero energy. The next-to-leading-order contribution $\propto 1 / \sqrt{\varepsilon}$ takes into account the small initial energy $E_{\text {in }}$. Finally, the last term $\propto \chi$ describes the displacement effect. 


\section{Steep incidence}

Turning the triangle around, we have for the scaled interior solution

$$
\psi(t, 0<x<L / \varepsilon)=\int d E e^{-i E t+i \varepsilon \varphi} \times\left\{\psi_{\text {int }}^{\mathrm{A}}(E) \operatorname{Ai}\left[g\left(\frac{L}{\varepsilon}-x+\chi\right)\right]+\psi_{\text {int }}^{\mathrm{B}}(E) \operatorname{Bi}\left[g\left(\frac{L}{\varepsilon}-x+\chi\right)\right]\right\} .
$$

We apply the same approximations as above and find from the junction conditions at $x=0$ the expansion coefficient

$$
\begin{aligned}
\psi_{\text {int }}^{A}(E)= & -\frac{2 i \sqrt{\pi} \sqrt{2 m \sqrt{\varepsilon} E_{\text {in }}}}{\left(2 m V_{0} \varepsilon / L\right)^{1 / 3}\left[\left(2 m V_{0} \varepsilon / L\right)^{1 / 3} L / \varepsilon\right]^{1 / 4}} \times \frac{1}{2 \pi} \int d t e^{i\left(E-E_{\text {in }}\right) t} \exp \left\{-\left(2 m V_{0}\right)^{1 / 2} \chi\left(\frac{t}{\sqrt{\varepsilon}}+i \frac{\mathfrak{T}}{\varepsilon}\right)\right\} \\
& \times \exp \left\{-\frac{2}{3}\left(2 m V_{0}\right)^{1 / 2} \frac{L}{\varepsilon}+\frac{E_{\text {in }} \mathfrak{T}}{\sqrt{\varepsilon}}\right\} .
\end{aligned}
$$

Here the steep slope at the front end of the triangle results in an energy mixing which is encoded in the complex time argument of the function $\chi$. Equation (A11) together with the junction conditions at the rear end of the triangle determine the transmission amplitude

$$
\begin{aligned}
\psi_{\text {trans }}(E)= & \exp \left\{-i \sqrt{2 m E \sqrt{\varepsilon}} \frac{L}{\varepsilon}+i\left(\frac{2}{3} z_{\mathrm{E}}^{3 / 2}-\frac{\pi}{4}\right)\right\} \times 2\left(\frac{\sqrt{\varepsilon} E_{\mathrm{in}}^{2}}{V_{0} E}\right)^{\frac{1}{4}} \int \frac{d t}{2 \pi} e^{i\left(E-E_{\mathrm{in}}\right) t} \\
& \times \exp \left\{-\frac{2}{3}\left(2 m V_{0}\right)^{1 / 2} \frac{L}{\varepsilon}+\frac{E_{\mathrm{in}} \mathfrak{T}}{\sqrt{\varepsilon}}+\left(2 m V_{0}\right)^{1 / 2} \chi\left(\frac{t}{\sqrt{\varepsilon}}+i \frac{\mathfrak{T}}{\varepsilon}\right)\right\} .
\end{aligned}
$$

Apart from a minor change $\left(E_{\mathrm{in}} / E\right)^{1 / 4}$ of the prefactor, the major difference between Eqs. (A12) and (A9) lies in the $\chi$ dependence of the exponential in the last line. The analytic continuation of the time-dependent function $\chi$ to complex time arguments reflects energy mixing (at the front end) and can enhance the tunneling amplitude strongly.

In contrast, the leading-order $\propto 1 / \varepsilon$ and next-to-leading-order $\propto 1 / \sqrt{\varepsilon}$ contributions to that exponential are the same in Eqs. (A12) and (A9). As a consistency check, the case of $\chi=0$ reproduces the time-independent solution of the Schrödinger equation where the $t$ integral in Eq. (A12) yields $\delta\left(E-E_{\mathrm{in}}\right)$ and thus the tunneling probabilities are the same.

\section{APPENDIX B: NUMERICAL SOLUTION TECHNIQUE}

The basic principle is to convert the time-dependent Schrödinger equation (2) into a system of coupled ordinary differential equations through the method of lines, where the coordinate variable $x$ is discretized beforehand and the resulting set of ordinary differential equations is then propagated in time. Derivatives in terms of $x$ are computed through pseudospectral methods whereas the time derivative is performed in terms of an explicit stepper (see Appendix B 1). The presence of a steep descent in the scalar potential $V(x)$ displaying the transition from a repulsive Coulomb potential to an attractive potential poses a special challenge which is discussed separately in Appendix B 2.

\section{Pseudospectral methods and time integration}

The main idea of pseudospectral methods is to compute all numerically cheap operations in the basic domain, but perform derivatives in the respective complementary domain where they become multiplications again $[73,74]$. For example, the kinetic operator can be formally written in terms of Fourier $\mathcal{F}$ and inverse Fourier transforms $\mathcal{F}^{-1}$ as

$$
\begin{aligned}
\partial_{x}^{2} \Psi(x) & =\mathcal{F}^{-1}\left\{\mathcal{F}\left\{\partial_{x}^{2} \Psi(x)\right\}\right\} \\
& =\mathcal{F}^{-1}\left\{\left(i k_{x}\right)^{2} \mathcal{F}\{\Psi(x)\}\right\} .
\end{aligned}
$$

In the context of assisted tunneling, boundary conditions are of no concern as we assume that the wave function falls off sufficiently fast in $x$ and, moreover, does not propagate beyond the limits of the spatial domain.
However, as the problem is posed as an initial-value problem, we do not know all temporal boundary conditions, thus, evaluating $\partial_{t}$ is not done spectrally. Instead, integration in time has been performed using a Dormand-Prince Runge-Kutta integrator of order $8(5,3)$. This is an adaptive, explicit, 12-step method of eighth order with embedded error correction of orders 5 and 3 [75]. This allows for continuous adjustments over the course of integration to ensure, e.g., norm preservation.

Errors due to the spatial discretization scale per $O\left([1 / N]^{N}\right)$ with the number of grid points $N$, while for the time stepping we have an error of order $O\left([\Delta t]^{8}\right)$.

For the sake of completeness, initial conditions in deuterium-tritium and proton-boron fusion are given by Gaussian wave packets of the form

$$
\begin{aligned}
\Psi(x, t=0)= & \frac{1}{\sqrt{\sqrt{2 \pi} \sigma}} \exp \left\{ \pm i \sqrt{2 m E_{\text {in }}}\left(x-a_{0}\right)\right\} \\
& \times \exp \left\{-\frac{\left(x-a_{0}\right)^{2}}{4 \sigma^{2}}\right\},
\end{aligned}
$$

with the particle's initial energy $E_{\text {in }}$, the initial peak location $a_{0}$, and the packet's initial width $\sigma$. Intricacies within muonassisted fusion are discussed in Appendix B 4.

\section{Discretizing the Coulomb potential}

In order to solve the Schrödinger equation by means of spectral methods we have to bring the vector potential in a form that allows an efficient evaluation in $x$ space without sacrificing accuracy in $k_{x}$ space. To this end we first perform a variable transformation in $x$ bringing the peak of the scalar 


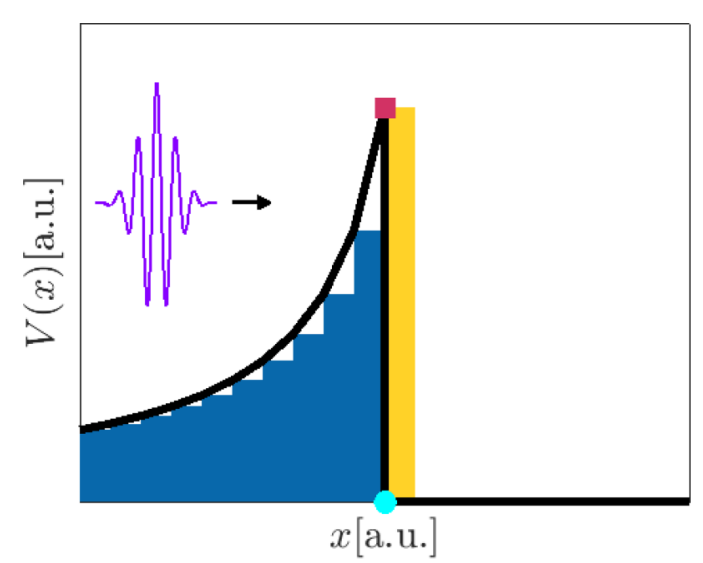

FIG. 4. Sketch of a lowest-order discretization scheme for a Coulomb potential with a steep gradient close to the nucleus. A wave propagating from left-to-right probes step functions (blue area: $\varepsilon<0$, yellow and blue area: $\varepsilon>0$ ) which become increasingly removed from the true value of the smooth Coulomb potential (black line). Only if the resolution is sufficiently high, the location of the grid point at the center (cyan circle: $\varepsilon<0$, purple square: $\varepsilon>0$ ) becomes irrelevant and the potentials coincide.

potential to the center of the domain

$$
V_{\text {num }}(x)=\frac{q_{1} q_{2}}{4 \pi \epsilon_{0}\left(|x|+\left|x_{\text {out }}\right|\right)} \Theta\left(-x+\varepsilon\left|x_{\text {out }}\right|\right)
$$

with the inner turning point $x_{\text {out }}<0$ given by the nuclear radius.

At this point we have to be very careful about the definition of the Heaviside function $\Theta$ as the steep dropoff of the potential cannot be represented exactly on a grid with finite resolution. Hence, we have to introduce a numerical parameter $\varepsilon$, which determines whether the minimal and maximal transmission rates are studied and thus serve as an indicator for convergence. In short, if the transmission rates for $1 \gg \varepsilon>0$ are reasonably close to the rates obtained for the same configuration but with $-\varepsilon$, we have reason to believe that spatial discretization is not a major source of error. Additionally, the resolution close to the peak of the potential is much more important than in the tail, thus, mappings in $x$ (in this work we used an exponential mapping) are advantageous as due to the increased point density at the origin the barrier can be better approximated. In Fig. 4 we have illustrated this problem.

Furthermore, for pseudospectral solvers a sudden change in the potential is problematic because it generally leads to instabilities and due to the Gibbs phenomenon oscillations in the wave function might emerge. These issues cannot be fully eliminated, but its effects can be minimized by performing multiple computations for a well-suited coordinate system and monitoring of the results for a gradual increase in, e.g., the number of grid points. In this way, outliers can be spotted easily, further increasing the robustness of the calculations.

\section{Attractive potential}

In contrast to the repulsive force exerted by the Coulomb potential, nuclear forces act attractive. However, the exact shape of the potential close to the nucleus is largely unknown. Furthermore, resolving any additional short-range force would require a much finer grid, leading to a significant increase in the total lattice size and, in turn, higher numerical cost. Hence, to not complicate matters further we opted for a simple, uniform potential well instead [76,77]

$$
V_{\text {nucl }}(x)=V_{0} \Theta\left(x-\varepsilon\left|x_{\text {out }}\right|\right),
$$

with $V_{0}<0$ and, again, the numerical parameter $\varepsilon$.

For the calculations regarding muon-assisted fusion, it is essential to include this nuclear attraction term because in this case, the initial state is a bound state with negative energy $E_{\text {in }}<0$ and thus tunneling would be impossible for $V_{0}=0$. For the other cases starting from an initial scattering state (B2) with positive energy $E_{\text {in }}>0$, this is no longer true and thus it is not necessary to include a nuclear potential $V_{0}$.

Nevertheless, to complete the picture, we studied how the tunneling probabilities change when lowering the nuclear potential $V_{0}$. We found that the impact of this nuclear potential $V_{0}$ does indeed modify the tunneling probabilities in both cases, with and without an electric field, but in nearly the same way, such that its effect on the enhancement has been found to be only of minor importance.

\section{Computing muon-assisted fusion}

As already stated in the main body of the text, muoncatalyzed fusion creates a three-body problem [71] that has to be solved under the influence of external sources. As such a study is well beyond the scope of this work, we employ a toy model (see, e.g., [68]). In this way we can fall back on solving the one-dimensional Schrödinger equation with a nonvanishing vector potential, a reduced mass $m$, and an effective charge $q_{\text {eff }}$. The main difference comes from the fact that, contrary to deuterium-tritium or proton-boron fusion, there is no incident wave packet. Instead, here the initial state is determined by a bound state.

In our toy model the bound state generated by the muon is represented by a deformation of the Coulomb potential in such a way that a pocket within the repulsive potential emerges. This muon-modified Coulomb potential can, for example, be described by

$$
V_{\text {bound }}(x)=\frac{q_{1} q_{2}}{4 \pi \epsilon_{0}}\left(\frac{1}{|x|+\left|x_{\text {out }}\right|}-\frac{\Delta V}{\left[1+\kappa\left(|x|+\left|x_{\text {out }}\right|\right)\right]^{4}}\right),
$$

where $\Delta V$ and $\kappa$ can be determined from the energy and length scales of the muon-generated bound state (cf. [70] for a discussion on this specific potential).

In terms of a numerical evaluation of muon-assisted fusion, we first consider a static situation where deuterium, tritium, and muon form a molecule. In order to have a well-posed problem, we demand that the wave function vanishes at $x=$ $x_{\text {out }}<0$. In this situation our effective model produces bound states describing a wave function that is trapped within the region $x \in\left(-\infty, x_{\text {out }}\right]$. Employing the shooting method the precise shape of the ground-state wave function is obtained, which, in turn, defines the initial state for the time-dependent Schrödinger solver.

In a second step, restrictions on the potential are lifted allowing the wave function to spread beyond this point, i.e., to $x>x_{\text {out }}$, and therefore to tunnel through the Coulomb barrier. 
TABLE II. Comparison of the enhancement and suppression of tunneling for various scenarios with the same values for energy $E_{\text {in }}=4 \mathrm{keV}$, pulse width $\omega=1 \mathrm{keV}$, and field strength $10^{16} \mathrm{~V} / \mathrm{m}$. "Incidence" refers to the slope of the potential at the entry point of the wave packet: Gradual or steep. Two pulse shapes were considered: a Sauter pulse $A_{0} / \cosh ^{2}(\omega t)$ and a Gaussian profile $A_{0} \exp \left\{-\omega^{2} t^{2}\right\} 2 / \sqrt{\pi}$, where the factor $2 / \sqrt{\pi}$ was chosen to keep the total displacement $\Delta \chi$ constant. The $A_{0}$ field was oriented parallel $(\rightarrow)$ or antiparallel $(\leftarrow)$ to the initial propagation velocity of the wave packet (i.e., the tunneling direction). The increase or decrease in the tunneling probability is given in terms of the ratio comparing the cases with and without field. The timing was chosen such that the vector potential peaks at the moment the peak of the wave packet makes contact with the barrier.

\begin{tabular}{lccc}
\hline \hline Incidence & Pulse shape & Field direction & Ratio \\
\hline Gradual & Sauter & $\leftarrow$ & 0.64 \\
& & $\rightarrow$ & 2.06 \\
& Gauss & $\leftarrow$ & 0.65 \\
Steep & Sauter & $\leftarrow$ & 2.18 \\
& & $\rightarrow$ & 0.65 \\
& Gauss & $\leftarrow$ & 3.02 \\
& & $\rightarrow$ & 0.71 \\
\hline \hline
\end{tabular}

Furthermore, the full potential exhibits also an attractive term

$$
V_{\text {muon }}=V_{\text {bound }}(x) \Theta\left(-x+\varepsilon\left|x_{\text {out }}\right|\right)+V_{\text {nucl }}(x) \text {. }
$$

The attractive potential $V_{0}$ is deemed to be uniform and, in view of accurate calculations, assumed to be $V_{0}=-10 \mathrm{keV}$. This value of $V_{0}$ is well below the bound-state energy and turned out to be a good compromise between creating a stimulus for the wave function to tunnel without sacrificing too much computational resources on a proper lattice discretization to account for a highly oscillating outwards propagating wave packet after tunneling.

\section{APPENDIX C: COULOMB RATCHETS}

For the triangular potential in Sec. IV, we have discussed the quantum ratchet effect, i.e., the dependence of enhancement or suppression of tunneling on the orientation of the potential. This dependence on the orientation of the potential is absent for stationary tunneling and thus a clear nonequilibrium phenomenon, which can be understood by the difference between the energy mixing and the displacement effects. As the Coulomb potential (cut off by the nuclear attraction) does also exhibit a strong asymmetry, one expects analogous effects in this case.

Table II presents the enhancement or suppression of tunneling (represented by the "ratio") in dependence of the orientation of the potential (i.e., the direction of "incidence") and the direction of the field. Furthermore, we studied the dependence on the pulse shape by comparing a Sauter pulse with a Gaussian profile. Even though these two pulse shapes look very similar as a function of real time $t \in \mathbb{R}$, their behavior in the complex $t$ plane is very different (see also [78]), which should affect the energy mixing effect.
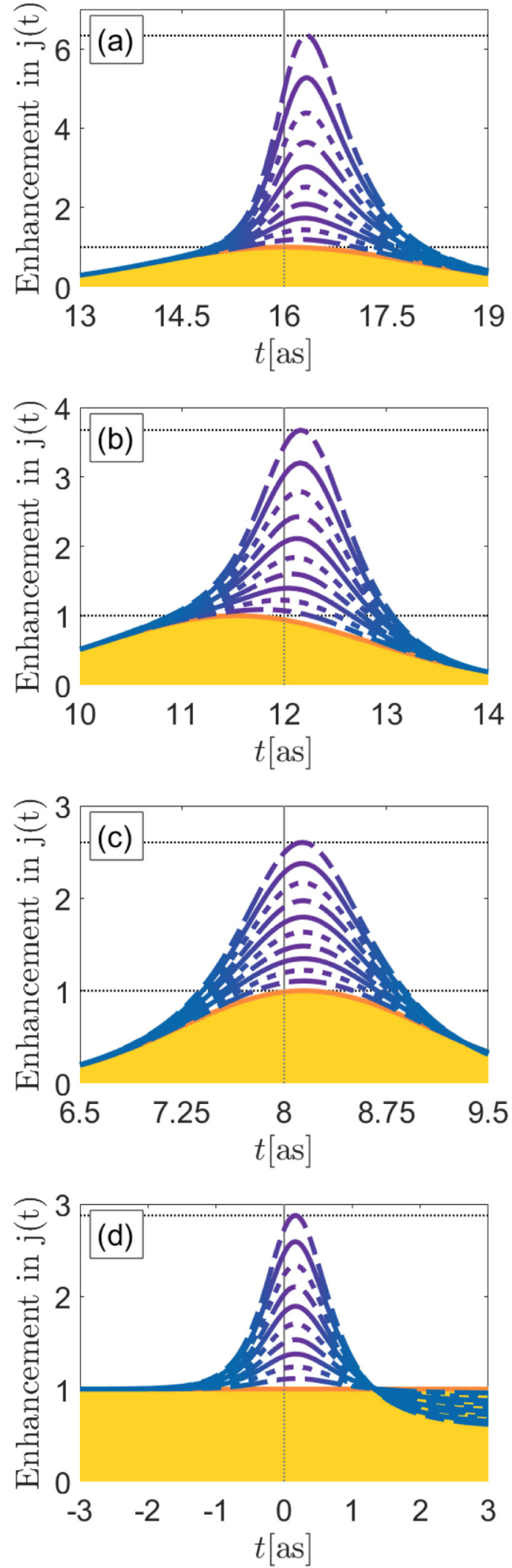

FIG. 5. Enhancement of the tunneling rates for the scenarios in Figs. 1 and 3 due to the pulse $A_{x}(t)$ with $\omega=1 \mathrm{keV}$ where the blue curves correspond to various peak field strengths $n \times 10^{15} \mathrm{~V} / \mathrm{m}$ with $n \in\{1,2,3, \ldots, 10\}$. 
The quantum ratchet effect is clearly visible in the differences between the upper and lower halves of Table II, especially for the enhancement of tunneling (for the parallel field direction). As expected, this enhancement is stronger for steep incidence, which we attribute to the higher efficiency of the energy mixing process.

This energy mixing process does also display a stronger dependence on the pulse shape (in view of the behavior in the complex plane or the Fourier transform, which yields the frequency spectrum of the pulse) than the displacement effect, for example, which is also visible in Table II. Note that analogous dependencies on the pulse shape have been discussed in the context of the dynamically assisted SauterSchwinger effect, which can also be understood as an example for dynamically assisted tunneling (see, e.g., [78]).

Finally, we observe, at least for the parameters used in Table II, a perfect correlation between the field direction and the enhancement or suppression of tunneling. For parallel fields (first accelerating and then decelerating the incident particle), tunneling is enhanced while antiparallel fields (where the free kinetic energy is lowered during the pulse) suppress tunneling. However, this intuitive picture is not always correct: As already stated above, the energy mixing process can also enhance tunneling for antiparallel fields if the spectral width of the pulse is broad enough to facilitate a sufficient upshift in energy, which, in turn, increases the tunneling probability more than its reduction due to the other processes. In fact, we observed the counterintuitive behavior numerically for a different set of parameters (pulse width $\omega=10 \mathrm{keV}$ and field strength $\left.10^{17} \mathrm{~V} / \mathrm{m}\right)$ in the case of antiparallel fields and steep incidence (as expected).

\section{APPENDIX D: FIELD-STRENGTH DEPENDENCE}

To conclude, let us briefly discuss the dependence of the enhancement on the peak field strength. Figure 5 displays the tunneling current $j(t)$ for the scenarios in Figs. 1 and 3 (i.e., deuterium-tritium fusion for initial kinetic energies of 2,4 , and $8 \mathrm{keV}$, as well as the case of muon-assisted fusion) for various peak field strengths $n \times 10^{15} \mathrm{~V} / \mathrm{m}$ with $n \in$ $\{1,2,3, \ldots, 10\}$. If we try to fit these field-strength dependencies of the maximum enhancement, we find that exponential fits (with different fitting factors in the exponent for the various cases) provide a very good description in all four scenarios.

Although this is very reminiscent of Eqs. (9) and (16), one should be careful regarding a direct identification. First, the results (9) and (16) were derived for idealized (rectangular and triangular) potentials and cannot be applied directly here. Second, other mechanisms such as preacceleration (i) and potential deformation (iii) can also induce an exponential dependence on the field strength. As explained above, all effects (i)-(iv) are intertwined for the parameters under consideration and cannot be disentangled easily. Their relative contributions may vary depending on the parameters and none of these effects (i)-(iv) alone can explain all of our findings.

For instance, a pulse $A_{x}(t)$ with $\omega=1 \mathrm{keV}$ and a peak field strength of $10^{16} \mathrm{~V} / \mathrm{m}$ can accelerate an effective particle (with $q_{\text {eff }}$ and $m$ ) at rest to a maximum kinetic energy of up to $0.1 \mathrm{keV}$. This preacceleration effect would not be sufficient to account for the enhancement seen in Fig. 3. Similarly, the static potential deformation with $10^{16} \mathrm{~V} / \mathrm{m}$ can also not explain this enhancement such that the nonadiabatic effects (ii) and/or (iv) must play a role.

To give a different example, the short Büttiker-Landauer traversal time of $\mathfrak{T} \approx 0.03$ as in proton-boron fusion at an initial kinetic energy of $80 \mathrm{keV}$ (see the bottom panel of Fig. 2) suggests that a pulse with $\omega=1 \mathrm{keV}$ is probably too slow to decisively probe nonadiabatic effects (ii) and (iv). Thus, adiabatic effects such as pre-acceleration (i) are expected to dominate in this case.

Disentangling the relative contributions of the various effects (i)-(iv) in dependence on the parameters should be the subject of future studies.
[1] Apart from the smallness of Planck's constant $\hbar$ in everyday units, the laws of quantum mechanics, such as the quantum Zeno effect $[79,80]$, prevent us from directly observing of the process of quantum tunneling. However, sometimes the evanescent tails in more general wave propagation phenomena are considered as analogs to tunneling. These could of course be observed with the naked eye, for example, in water waves.

[2] I. Bloch, Ultracold quantum gases in optical lattices, Nat. Phys. 1, 23 (2005).

[3] O. Morsch and M. Oberthaler, Dynamics of Bose-Einstein condensates in optical lattices, Rev. Mod. Phys. 78, 179 (2006).

[4] G. Gamow, Zur Quantentheorie des Atomkernes, Z. Phys. 51, 204 (1928).

[5] H. Geiger and J. M. Nuttall, The ranges of the $\alpha$ particles from various radioactive substances and a relation between range and period of transformation, Philos. Mag. Ser. 22, 613 (1911); The ranges of the $\alpha$ particles from uranium, 23, 439 (1912).

[6] R. Gurney and E. Condon, Wave mechanics and radioactive disintegration, Nature (London) 122, 439 (1928).
[7] As one example, let us mention the recent controversy regarding the dynamical assistance of tunneling in nuclear $\alpha$ decays (see, e.g., [45] and Sec. VE). Another example is the ongoing discussion about the tunneling time (see, e.g., [21,22] and Sec. V A).

[8] S. Coleman, The uses of instantons, in Aspects of Symmetry (Cambridge University Press, Cambridge, 1985).

[9] M. Grifoni and P. Hänggi, Driven quantum tunneling, Phys. Rep. 304, 229 (1998).

[10] M. Razavy, Quantum Theory of Tunneling (World Scientific, Singapore, 2003).

[11] W. Franz, Einfluß eines elektrischen Feldes auf eine optische Absorptionskante, Z. Naturforsch. A 13, 484 (1958).

[12] L. V. Keldysh, The effect of a strong electric field on the optical properties of insulating crystals, J. Exper. Theor. Phys. 34, 1138 (1958) [Sov. Phys.-JETP 7, 788 (1958)].

[13] W. C. Henneberger, Perturbation Method for Atoms in Intense Light Beams, Phys. Rev. Lett. 21, 838 (1968). 
[14] M. Büttiker and R. Landauer, Traversal Time for Tunneling, Phys. Rev. Lett. 49, 1739 (1982).

[15] More details regarding the applicability of the instanton picture to dynamically assisted tunneling will be postponed to a forthcoming publication.

[16] F. Queisser and R. Schützhold, Dynamically assisted nuclear fusion, Phys. Rev. C 100, 041601(R) (2019).

[17] B. I. Ivlev and V. I. Melńikov, Dramatic stimulation of tunneling by an rf field, Pis'ma Zh. Eksp. Teor. Fiz. 41, 116 (1985) [JETP Lett. 41, 142 (1985)].

[18] P. Reimann, M. Grifoni, and P. Hänggi, Quantum Ratchets, Phys. Rev. Lett. 79, 10 (1997).

[19] H. Linke, T. E. Humphrey, A. Löfgren, A. O. Sushkov, R. Newbury, R. P. Taylor, and P. Omling, Experimental tunneling ratchets, Science 286, 2314 (1999).

[20] A. S. Landsman, M. Weger, J. Maurer, R. Boge, A. Ludwig, S. Heuser, C. Cirelli, L. Gallmann, and U. Keller, Ultrafast resolution of tunneling delay time, Optica 1, 343 (2014).

[21] R. Ramos, D. Spierings, I. Racicot, and A. M. Steinberg, Measurement of the time spent by a tunnelling atom within the barrier region, Nature (London) 583, 529 (2020).

[22] H. G. Winful, Tunneling time, the Hartman effect, and superluminality: A proposed resolution of an old paradox, Phys. Rep. 436, 1 (2006).

[23] C. Sias, H. Lignier, Y. P. Singh, A. Zenesini, D. Ciampini, O. Morsch, and E. Arimondo, Observation of Photon-Assisted Tunneling in Optical Lattices, Phys. Rev. Lett. 100, 040404 (2008).

[24] R. Ma, M. E. Tai, P. M. Preiss, W. S. Bakr, J. Simon, and M. Greiner, Photon-Assisted Tunneling in a Biased Strongly Correlated Bose Gas, Phys. Rev. Lett. 107, 095301 (2011).

[25] Q. Niu, X.-G. Zhao, G. A. Georgakis, and M. G. Raizen, Atomic Landau-Zener Tunneling and Wannier-Stark Ladders in Optical Potentials, Phys. Rev. Lett. 76, 4504 (1996).

[26] F. Queisser, P. Navez, and R. Schützhold, Sauter-Schwingerlike tunneling in tilted Bose-Hubbard lattices in the Mott phase, Phys. Rev. A 85, 033625 (2012).

[27] A. Eckardt, Colloquium: Atomic quantum gases in periodically driven optical lattices, Rev. Mod. Phys. 89, 011004 (2017).

[28] E. O. Kane, Zener tunneling in semiconductors, J. Phys. Chem. Solids 12, 181 (1959).

[29] E. O. Kane, Theory of tunneling, J. Appl. Phys. 32, 83 (1961).

[30] C. Zener, A theory of the electrical breakdown of solid dielectrics, Proc. R. Soc. Lond. A 145, 523 (1934).

[31] W. Vandenberghe, B. Sorée, W. Magnus, and G. Groeseneken, Zener tunneling in semiconductors under nonuniform electric fields, J. Appl. Phys. 107, 054520 (2010).

[32] B. G. Streetman and S. Banerjee, Solid State Electronic Devices (Prentice Hall, Englewood Cliffs, NJ, 2005).

[33] R. F. Voss and R. A. Webb, Macroscopic Quantum Tunneling in 1- $\mu m$ Nb Josephson Junctions, Phys. Rev. Lett. 47, 265 (1981).

[34] M. F. Linder, A. Lorke, and R. Schützhold, Analog SauterSchwinger effect in semiconductors for spacetime-dependent fields, Phys. Rev. B 97, 035203 (2018).

[35] S. Augst, D. Strickland, D. D. Meyerhofer, S. L. Chin, and J. H. Eberly, Tunneling Ionization of Noble Gases in a High-Intensity Laser Field, Phys. Rev. Lett. 63, 2212 (1989); Erratum: "Tunneling ionization of noble gases in a high-intensity laser field” [Phys. Rev. Lett. 63, 2212 (1989)] 66, 1247(E) (1991).
[36] A. Hartung, F. Morales, M. Kunitski, K. Henrichs, A. Laucke, M. Richter, T. Jahnke, A. Kalinin, M. Schöffler, L. Ph. H. Schmidt et al., Electron spin polarization in strong-field ionization of xenon atoms, Nat. Photonics 10, 526 (2016).

[37] C. A. Mancuso, D. D. Hickstein, P. Grychtol, R. Knut, O. Kfir, X. M. Tong, F. Dollar, D. Zusin, M. Gopalakrishnan, C. Gentry et al., Strong-field ionization with two-color circularly polarized laser fields, Phys. Rev. A 91, 031402(R) (2015).

[38] L. V. Keldysh, Ionization in the field of a strong electromagnetic wave, J. Exptl. Theoret. Phys. (U.S.S.R.) 47, 1945 (1964) [Sov. Phys.-JETP 20, 1307 (1965)].

[39] P. B. Corkum, Plasma Perspective on Strong Field Multiphoton Ionization, Phys. Rev. Lett. 71, 1994 (1993).

[40] V. S. Popov, Tunnel and multiphoton ionization of atoms and ions in a strong laser field (Keldysh theory), Phys.-Usp. 47, 855 (2004).

[41] P. Agostini, F. Fabre, G. Mainfray, G. Petite, and N. K Rahman, Free-Free Transitions Following Six-Photon Ionization of Xenon Atoms, Phys. Rev. Lett. 42, 1127 (1979).

[42] S. L. Chin, F. Yergeau, and P. Lavigne, Tunnel ionisation of Xe in an ultra-intense $\mathrm{CO}_{2}$ laser field $\left(10^{14} \mathrm{~W} \mathrm{~cm}^{-2}\right)$ with multiple charge creation, J. Phys. B: At. Mol. Phys. 18, L213 (1985).

[43] Th. Stöhlker, P. H. Mokler, F. Bosch, R. W. Dunford, F. Franzke, O. Klepper, C. Kozhuharov, T. Ludziejewski, F. Nolden, H. Reich et al., 1s Lamb Shift in Hydrogenlike Uranium Measured on Cooled, Decelerated Ion Beams, Phys. Rev. Lett. 85, 3109 (2000).

[44] D. S. Delion and S. A. Ghinescu, Geiger-Nuttall Law for Nuclei in Strong Electromagnetic Fields, Phys. Rev. Lett. 119, 202501 (2017).

[45] A. Pálffy and S. V. Popruzhenko, Can Extreme Electromagnetic Fields Accelerate the $\alpha$ Decay of Nuclei? Phys. Rev. Lett. 124, 212505 (2020).

[46] C. Schneider and R. Schützhold, Dynamically assisted SauterSchwinger effect in inhomogeneous electric fields, J. High Energy Phys. 02 (2016) 164.

[47] N. G. Kelkar, H. M. Castañeda, and M. Nowakowski, Quantum time scales in alpha tunneling, Europhys. Lett. 85, 20006 (2009).

[48] B. Ivlev and V. Gudkov, New enhanced tunneling in nuclear processes, Phys. Rev. C 69, 037602 (2004).

[49] W. Lv, H. Duan, and J. Liu, Enhanced deuterium-tritium fusion cross sections in the presence of strong electromagnetic fields, Phys. Rev. C 100, 064610 (2019).

[50] F. Queisser and R. Schützhold, Comment on Enhanced deuterium-tritium fusion cross sections in the presence of strong electromagnetic field, arXiv:2003.02661.

[51] G. Kälbermann, Assisted fusion, arXiv:0910.3447.

[52] M. L. E. Oliphant and E. Rutherford, Experiments on the transmutation of elements by protons, Proc. R. Soc. London, Ser. A 141, 259 (1933).

[53] JET Team, Fusion energy production from a deuterium-tritium plasma in the JET tokamak, Nucl. Fusion 32, 187 (1992).

[54] K. M. McGuire, H. Adler, P. Alling, C. Ancher, H. Anderson, J. L. Anderson, J. W. Anderson, V. Arunasalam, G. Ascione, D. Ashcroft et al., Review of deuterium-tritium results from the Tokamak Fusion Test Reactor, Phys. Plasmas 2, 2176 (1995).

[55] L. Giuffrida, F. Belloni, D. Margarone, G. Petringa, G. Milluzzo, V. Scuderi, A. Velyhan, M. Rosinski, A. Picciotto, M. Kucharik, J. Dostal et al., High-current stream of energetic 
$\alpha$ particles from laser-driven proton-boron fusion, Phys. Rev. E 101, 013204 (2020).

[56] D. C. Moreau, Potentiality of the proton-boron fuel for controlled thermonuclear fusion, Nucl. Fusion 17, 13 (1977).

[57] H. Hora, G. H. Miley, M. Ghoranneviss, B. Malekynia, N. Azizi, and X.-T. He, Fusion energy without radioactivity: laser ignition of solid hydrogen-boron (11) fuel, Energy Environ. Sci. 3, 478 (2010).

[58] C. Labaune, C. Baccou, S. Depierreux, C. Goyon, G. Loisel, V. Yahia, and J. Rafelski, Fusion reactions initiated by laseraccelerated particle beams in a laser-produced plasma, Nat. Commun. 4, 2506 (2013).

[59] S. Gordienko, A. Pukhov, O. Shorokhov, and T. Baeva, Coherent Focusing of High Harmonics: A New Way Towards the Extreme Intensities, Phys. Rev. Lett. 94, 103903 (2005).

[60] H. Vincenti, Achieving Extreme Light Intensities Using Optically Curved Relativistic Plasma Mirrors, Phys. Rev. Lett. 123, 105001 (2019).

[61] L. Fedeli, A. Sainte-Marie, N. Zaïm, M. Thévenet, J. L. Vay, A. Myers, F. Quéré, and H. Vincenti, Probing strong-field QED with Doppler-boosted PetaWatt-class lasers, arXiv:2012.07696.

[62] G. A. Mourou, T. Tajima, and S. V. Bulanov, Optics in the relativistic regime, Rev. Mod. Phys. 78, 309 (2006).

[63] In the presence of the Coulomb field of an additional nucleus (such as an $\alpha$ particle) depending on both space and time, the split into center-of-mass and relative coordinates in Sec. VI A as well as the Kramers-Henneberger map in Sec. II cannot be applied in this way. Furthermore, in contrast to a purely timedependent electric field with a fixed direction, the direction of the Coulomb field of an additional nucleus (incident at an impact parameter of order $500 \mathrm{fm}$ or below and under a finite angle to the tunneling direction) changes in time, which invalidates a one-dimensional treatment. Finally, a complete analysis would require solving the full three-body problem (e.g., deuterium and tritium nuclei as well as the $\alpha$ particle) which is a complex and intricate task.

[64] C. Kohlfürst, Effect of time-dependent inhomogeneous magnetic fields on the particle momentum spectrum in electron-positron pair production, Phys. Rev. D 101, 096003 (2020).

[65] I. A. Aleksandrov, G. Plunien, and V. M. Shabaev, Dynamically assisted Schwinger effect beyond the spatially-uniform-field approximation, Phys. Rev. D 97, 116001 (2018).
[66] R. Schützhold, H. Gies, and G. Dunne, Dynamically Assisted Schwinger Mechanism, Phys. Rev. Lett. 101, 130404 (2008).

[67] F. Frank, Hypothetical alternative energy sources for the 'Second Meson' Events, Nature (London) 160, 525 (1947).

[68] L. W. Alvarez, H. Bradner, F. S. Crawford, Jr., J. A. Crawford, P. Falk-Vairant, M. L. Good, J. D. Gow, A. H. Rosenfeld, F. Solmitz, M. L. Stevenson et al., Catalysis of nuclear reactions by $\mu$ mesons, Phys. Rev. 105, 1127 (1957).

[69] L. I. Ponomarev, Muon catalysed fusion, Contemp. Phys. 31, 219 (1990).

[70] J. D. Jackson, Catalysis of nuclear reactions between hydrogen isotopes by $\mu^{-}$mesons, Phys. Rev. 106, 330 (1957).

[71] V. S. Melezhik, L. I. Ponomarev, and M. P. Faifman, Elastic scattering in a three-particle system with Coulomb interaction, Zh. Eksp. Teor. Fiz. 85, 434 (1983) [Soviet Physics - JETP 58, 254 (1983)].

[72] D. V. Balin, V. A. Ganzha, S. M. Kozlov, E. M. Maev, G. E. Petrov, M. A. Soroka, G. N. Schapkin, G. G. Semenchuk, V. A. Trofimov, A. A. Vasiliev et al., High precision study of muon catalyzed fusion in $D_{2}$ and HD gas, Phys. Part. Nucl. 42, 185 (2011).

[73] J. P. Boyd, Chebyshev and Fourier Spectral Methods (Dover, New York, 2001).

[74] M. Frigo and S. G. Johnson, The design and implementation of FFTW3, Proc. IEEE 93, 216 (2005).

[75] W. H. Press, S. A. Teukolsky, W. T. Vetterling, and B. P. Flannery, Numerical Recipes 3rd Edition: The Art of Scientific Computing (Cambridge University Press, Cambridge, 2007).

[76] H. Feshbach, C. E. Porter, and V. F. Weisskopf, The formation of a compound nucleus in neutron reactions, Phys. Rev. 90, 166 (1953).

[77] R. K. Adair, Nuclear potential well depth, Phys. Rev. 94, 737 (1954).

[78] M. F. Linder, C. Schneider, J. Sicking, N. Szpak, and R. Schützhold, Pulse shape dependence in the dynamically assisted Sauter-Schwinger effect, Phys. Rev. D 92, 085009 (2015).

[79] B. Misra and E. C. G. Sudarshan, The Zeno's paradox in quantum theory, J. Math. Phys. 18, 756 (1977).

[80] W. M. Itano, D. J. Heinzen, J. J. Bollinger, and D. J. Wineland, Quantum Zeno effect, Phys. Rev. A 41, 2295 (1990). 